\title{
Koszul complexes and symmetric forms over the punctured affine
}

\section{space}

\section{Journal Article}

Author(s):

Balmer, Paul; Gille, Stefan

Publication date:

2005

Permanent link:

https://doi.org/10.3929/ethz-b-000031324

Rights / license:

In Copyright - Non-Commercial Use Permitted

Originally published in:

Proceedings of the London Mathematical Society 91(2), https://doi.org/10.1112/S0024611504015199 


\section{KOSZUL COMPLEXES AND SYMMETRIC FORMS OVER THE PUNCTURED AFFINE SPACE}

\section{PAUL BALMER AND STEFAN GILLE}

\section{Introduction}

Let $X$ be a scheme. We are studying the (total) graded Witt ring

$$
\mathrm{W}^{\mathrm{tot}}(X):=\bigoplus_{i \in \mathbb{Z}} \mathrm{W}^{i}(X)
$$

where the groups $\mathrm{W}^{i}$ are the derived Witt groups of Balmer $[\mathbf{2}, \mathbf{3}]$ with the multiplicative structure of Gille and Nenashev [11]. See more in $\S 3$.

We fix an integer $n \geqslant 1$ for the entire article. Consider the following open subset $\mathbb{U}_{\mathbb{Z}}^{n} \subset \mathbb{A}_{\mathbb{Z}}^{n}$ of the affine space $\mathbb{A}_{\mathbb{Z}}^{n}=\operatorname{Spec}\left(\mathbb{Z}\left[T_{1}, \ldots, T_{n}\right]\right)$ :

$$
\mathbb{U}_{\mathbb{Z}}^{n}:=\bigcup_{j=1}^{n} \operatorname{Spec}\left(\mathbb{Z}\left[T_{1}, \ldots, T_{n}, T_{j}^{-1}\right]\right) \subset \mathbb{A}_{\mathbb{Z}}^{n} .
$$

For any scheme $X$, define by base-change the open subscheme $\mathbb{U}_{X}^{n} \subset \mathbb{A}_{X}^{n}$, called the punctured affine space over $X$, that is, define $\mathbb{U}_{X}^{n}$ to be the following pull-back:

$$
\mathbb{U}_{X}^{n}:=X \times \mathbb{U}_{\mathbb{Z}}^{n}
$$

Our main results are summarized in Theorem 9.13 below, which is as follows.

Theorem. If $X$ is regular, contains $\frac{1}{2}$ and has finite Krull dimension, there is a decomposition $\mathrm{W}^{\mathrm{tot}}\left(\mathbb{U}_{X}^{n}\right)=\mathrm{W}^{\text {tot }}(X) \oplus \mathrm{W}^{\text {tot }}(X) \cdot \varepsilon$ for some Witt class $\varepsilon=\varepsilon_{X}^{(n)}$ in $\mathrm{W}^{n-1}\left(\mathbb{U}_{X}^{n}\right)$. If $n=1$, we have $\varepsilon^{2}=1$. If $n \geqslant 2$, we have $\varepsilon^{2}=0$ and an isomorphism

$$
\mathrm{W}^{\mathrm{tot}}\left(\mathbb{U}_{X}^{n}\right) \cong \frac{\mathrm{W}^{\mathrm{tot}}(X)[\varepsilon]}{\varepsilon^{2}}
$$

of graded rings, with the generator $\varepsilon$ in degree $n-1$.

When $n=1$, and at least for $X$ affine, $\mathbb{U}_{X}^{n}$ consists of the 'Laurent polynomials' over $X$, in which case the above theorem is due to Ranicki [16]; see also [15]. Note that the decomposition of $\mathrm{W}^{\text {tot }}\left(\mathbb{U}_{X}^{n}\right)$ for $n=1$ as two copies of $\mathrm{W}^{\text {tot }}(X)$ remains true for $n \geqslant 2$, but that the second copy is shifted by $n-1$, which is of course not visible when $n=1$. Note also that the ring structure is different from the Laurent case when $n \geqslant 2$. The special case $n=1$ also shows that this result cannot hold in general for non-regular schemes; see $[\mathbf{1 5}, \S 8]$ by Ojanguren and Panin.

The second goal of the article is an explicit description of the Witt class $\varepsilon_{X}^{(n)} \in \mathrm{W}^{n-1}\left(\mathbb{U}_{X}^{n}\right)$. It is constructed as the Witt class of a symmetric space denoted $\mathbf{E}_{X}^{(n)}=\left(\mathrm{E}_{X}^{(n)}, \xi_{X}^{(n)}\right)$, which exists for any scheme $X$, not necessarily regular. Here $\mathrm{E}_{X}^{(n)}$ is

Received 9 January 2004; revised 9 September 2004.

2000 Mathematics Subject Classification 11E81, $19 \mathrm{G} 12$.

Both authors are supported by the Swiss National Science Foundation, grant 620-066065. 
a complex in the derived category of vector bundles over $\mathbb{U}_{X}^{n}$ and the form $\xi_{X}^{(n)}$ is a symmetric quasi-isomorphism. Both are constructed via a suitable truncation of the Koszul complex over $\mathbb{A}_{X}^{n}$ for the regular sequence $\left(T_{1}, \ldots, T_{n}\right)$. This explicit description is necessary to prove the following fact (see Theorem 9.10).

Theorem. Assume $n \geqslant 2$. The symmetric space $\mathbf{E}_{X}^{(n)}$ is locally metabolic on $\mathbb{U}_{X}^{n}$.

This result does not hold for $n=1$ and is used to establish the announced relation $\left(\varepsilon_{X}^{(n)}\right)^{2}=0$; see Theorem 9.12. On the other hand, Theorem 10.2 says the following.

Theorem. The symmetric space $\mathbf{E}_{X}^{(n)}$ cannot be extended from $\mathbb{U}_{X}^{n}$ to $\mathbb{A}_{X}^{n}$, not even up to Witt equivalence. In particular, $\mathbf{E}_{X}^{(n)}$ is not metabolic on $\mathbb{U}_{X}^{n}$.

In other words, this symmetric space $\mathbf{E}_{X}^{(n)}$ is a little miracle happening over $\mathbb{U}_{X}^{n}$, which vanishes when restricted to smaller open subschemes and which cannot be extended to the bigger scheme $\mathbb{A}_{X}^{n}$.

Our last goal is a description of the generator $\varepsilon_{X}^{(n)} \in \mathrm{W}^{n-1}\left(\mathbb{U}_{X}^{n}\right)$ in 'classical' terms. Recall a few facts. First, the derived Witt groups are 4 -periodic: $\mathrm{W}^{i}=\mathrm{W}^{i+4}$. Secondly, $\mathrm{W}^{0}$ and $\mathrm{W}^{2}$ are naturally isomorphic to the usual Witt groups $\mathrm{W}_{\mathrm{us}}^{+}$and $\mathrm{W}_{\mathrm{us}}^{-}$ of symmetric and skew-symmetric vector bundles respectively, as defined by Knebusch [14]. Thirdly, $\mathrm{W}^{1}$ and $\mathrm{W}^{3}=\mathrm{W}^{-1}$ are groups of formations; see Walter [18]. So, describing 'in classical terms' our generator $\varepsilon_{X}^{(n)}$ in $\mathrm{W}^{n-1}$ amounts to producing an explicit element of the above nature, that is, a \pm 1 -symmetric form or formation, depending on the congruence of $n$ modulo 4 . This short symmetric space is denoted $\mathbf{F}_{X}^{(n)}$ and appears in $\S 8$.

There are two appendices. In the first one, we show that when $n \geqslant 4$ our locally free $\mathcal{O}_{\mathbb{U}_{X}^{n}}$-module $\mathcal{E}_{X}^{(n)}$ cannot be extended to a locally free $\mathcal{O}_{\mathbb{A}_{X}^{n}}$-module and in particular $\mathcal{E}_{X}^{(n)}$ is not free. The second appendix contains the compatibility between product and 4-periodicity, a fact which we use several times in this work.

\section{Conventions and notation}

We collect here the notation which is kept unchanged in all sections.

First, recall that we have fixed an integer $n \geqslant 1$. We decompose it as

$$
n=4 q+r+1
$$

where $q \in \mathbb{N}$ and $r \in\{-1,0,1,2\}$. Note that $n-1 \equiv r \bmod 4$. We also baptize

$$
\left[\frac{n}{2}\right]=: \ell .
$$

Convention 2.1. Unless otherwise mentioned, a ring means a commutative ring with unit.

Convention 2.2. As always, when using a notation defined for schemes $X$ in the affine case, $X=\operatorname{Spec}(R)$, we shall drop 'Spec' as, for instance: $\mathrm{VB}_{R}, \mathbb{D}^{\mathrm{b}}\left(\mathrm{VB}_{R}\right)$, $\mathrm{W}^{i}(R)$ instead of $\operatorname{VB}_{\mathrm{Spec}(R)}, \mathbb{D}^{\mathrm{b}}\left(\mathrm{VB}_{\mathrm{Spec}(R)}\right), \mathrm{W}^{i}(\operatorname{Spec}(R))$, and so on. See also Remark 3.3. 
Convention 2.3. We shall say that a scheme is regular if it is noetherian and separated and if all its local rings are regular.

Notation 2.4. Let $f: Y \rightarrow X$ be a morphism of schemes. We denote by $\mathbb{A}_{X}^{n}$ the affine $n$-space and by $\mathbb{U}_{X}^{n}$ the punctured affine $n$-space as in the introduction. The obvious structure morphisms and base-change morphisms will be denoted:

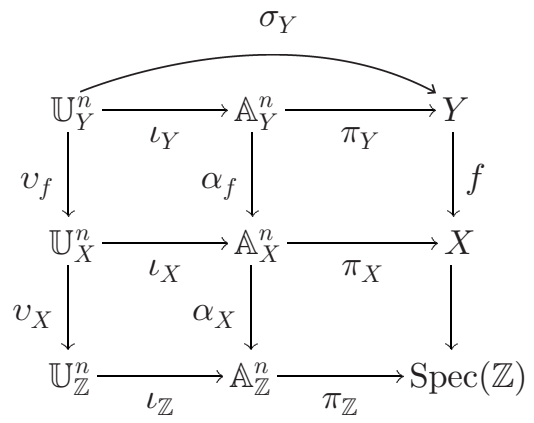

\section{Recalling derived Witt groups}

This section is a quick course on triangular Witt groups over schemes, included only for the reader's convenience. Here, $X$ is a scheme with structure bundle $\mathcal{O}_{X}$.

\subsection{Categories and dualities}

We denote by the symbol $\mathrm{VB}_{X}$ the exact category of locally free $\mathcal{O}_{X}$-modules of finite rank, that is, vector bundles. The usual duality on $\mathrm{VB}_{X}$ is abbreviated

$$
(-)^{\vee}:=\mathcal{H}^{\circ o m} \mathcal{O}_{X}\left(-, \mathcal{O}_{X}\right) \text {. }
$$

Here $\mathbb{D}^{\mathrm{b}}\left(\mathrm{VB}_{X}\right)$ stands for the bounded derived category of $\mathrm{VB}_{X}$. We use homological notation for complexes. The translation functor $\Sigma: \mathbb{D}^{\mathrm{b}}\left(\mathrm{VB}_{X}\right) \rightarrow \mathbb{D}^{\mathrm{b}}\left(\mathrm{VB}_{X}\right)$, also written $P_{\bullet} \mapsto P_{\bullet}[1]$, is given by $\left(P_{\bullet}[1]\right)_{j}=P_{j-1}$; as usual, $\Sigma$ changes the sign of all differentials: $d_{j}^{P[1]}=-d_{j-1}^{P}$.

Let $P_{\bullet}=\left(P_{\bullet}, d_{\bullet}^{P}\right)$ be a complex in $\mathbb{D}^{\mathrm{b}}\left(\mathrm{VB}_{X}\right)$. Its dual $\mathcal{D}_{X}\left(P_{\bullet}\right)$ is the complex

$$
\begin{aligned}
& \mathcal{D}_{X}\left(P_{\bullet}\right):=\quad \cdots \longrightarrow P_{-j} \vee \stackrel{d_{-j+1}^{P} \vee}{\longrightarrow} P_{-(j-1)} \longrightarrow \\
& \operatorname{deg} j \quad \operatorname{deg}(j-1)
\end{aligned}
$$

and similarly for morphisms of complexes. In other words, $\mathcal{D}_{X}$ is the derived functor of $(-)^{\vee}=\mathcal{H} m_{\mathcal{O}_{X}}\left(-, \mathcal{O}_{X}\right)$. This defines a 1-exact duality on $\mathbb{D}^{\mathrm{b}}\left(\mathrm{VB}_{X}\right)$ turning it into a triangulated category with duality in the sense of [2]. Recall that '1-exact' means that the dual of an exact triangle

$$
N_{.} \stackrel{\rho}{\longrightarrow} P . \stackrel{\phi}{\longrightarrow} Q . \stackrel{\varphi}{\longrightarrow} N .[1]
$$

is given by

$$
\mathcal{D}_{X}\left(Q_{\bullet}\right) \stackrel{\mathcal{D}_{X}(\phi)}{\longrightarrow} \mathcal{D}_{X}(P .) \stackrel{\mathcal{D}_{X}(\rho)}{\longrightarrow} \mathcal{D}_{X}\left(N_{\bullet}\right) \stackrel{\mathcal{D}_{X}(\varphi)[1]}{\longrightarrow} \mathcal{D}_{X}\left(Q_{\bullet}\right)[1] .
$$


The isomorphism between the identity and the double dual,

$$
\varpi: \operatorname{id}_{\mathbb{D}^{\mathrm{b}}\left(\mathrm{VB}_{X}\right)} \stackrel{\simeq}{\longrightarrow} \mathcal{D}_{X} \mathcal{D}_{X}
$$

is given in each degree $j$ by the canonical (evaluation) isomorphism

$$
\operatorname{can}_{P_{j}}: P_{j} \longrightarrow P_{j}^{\vee v} \text {. }
$$

We consider $\mathrm{VB}_{X}$ as a subcategory of $\mathbb{D}^{\mathrm{b}}\left(\mathrm{VB}_{X}\right)$ via the natural embedding $\mathrm{VB}_{X} \rightarrow \mathbb{D}^{\mathrm{b}}\left(\mathrm{VB}_{X}\right)$, which we denote $c_{0}$. The restriction of the duality $\mathcal{D}_{X}$ to this subcategory is the original duality of $\mathrm{VB}_{X}$ and the restriction of $\varpi$ is the above isomorphism can.

Definition 3.1. Let $P$. be a complex in $\mathbb{D}^{\mathrm{b}}\left(\mathrm{VB}_{X}\right)$. Let $i \in \mathbb{Z}$, and let $\phi: P_{\bullet} \rightarrow \mathcal{D}_{X}\left(P_{\bullet}\right)[i]$ be a morphism in $\mathbb{D}^{\mathrm{b}}\left(\mathrm{VB}_{X}\right)$. We say that $\phi$ is a symmetric $i$-form on the complex $P$. if

$$
\mathcal{D}_{X}(\phi)[i] \cdot \varpi_{P_{\bullet}}=(-1)^{i(i+1) / 2} \phi .
$$

We then say that $\left(P_{\bullet}, \phi\right)$ is a symmetric $i$-pair. If $\phi$ is moreover an isomorphism, we say that $\left(P_{\bullet}, \phi\right)$ is a symmetric $i$-space over $X$. Two symmetric $i$-pairs $(P ., \phi)$ and $(Q ., \psi)$ are called isometric if there exists in $\mathbb{D}^{\mathrm{b}}\left(\mathrm{VB}_{X}\right)$ an isometry between them, that is, an isomorphism $h: P \stackrel{\simeq}{\rightarrow} Q$. such that $\phi=\mathcal{D}_{X}(h)[i] \cdot \psi \cdot h$.

REMARK 3.2. Note that if $\left(P_{\bullet}, \phi\right)$ is a symmetric $i$-pair then $(P .[2], \phi[2])$ is a symmetric $(i+4)$-pair because $\mathcal{D}_{X}\left(P_{\bullet}\right)[1]=\mathcal{D}_{X}\left(P_{\bullet}[-1]\right)$ for all $P_{\bullet} \in \mathbb{D}^{\mathrm{b}}\left(\mathrm{VB}_{X}\right)$.

Let $f: Y \rightarrow X$ be a morphism of schemes. There is a natural isomorphism of functors $\eta_{f}: f^{*} \mathcal{D}_{X} \stackrel{\simeq}{\rightarrow} \mathcal{D}_{Y} f^{*}$ which is induced by the natural isomorphism of locally free $\mathcal{O}_{Y}$-modules

$$
f^{*} \mathcal{H o m}_{\mathcal{O}_{X}}\left(P, \mathcal{O}_{X}\right) \stackrel{\simeq}{\longrightarrow} \mathcal{H o m}_{\mathcal{O}_{Y}}\left(f^{*} P, \mathcal{O}_{Y}\right)
$$

If now $(P ., \phi)$ is a symmetric $i$-space over $X$ then the isomorphism

$$
f^{*}\left(P_{\bullet}\right) \stackrel{f^{*} \phi}{\longrightarrow} f^{*}\left(\mathcal{D}_{X}\left(P_{\bullet}\right)[i]\right)=f^{*}\left(\mathcal{D}_{X}\left(P_{\bullet}\right)\right)[i] \stackrel{\eta_{f, P}[i]}{\longrightarrow} \mathcal{D}_{Y}\left(f^{*} P_{\bullet}\right)[i]
$$

is a symmetric $i$-form and so $f^{*}\left(P_{\bullet}, \phi\right):=\left(f^{*}\left(P_{\bullet}\right), \eta_{f, P}[i] \cdot f^{*}(\phi)\right)$ is a symmetric $i$-space over $Y$.

\section{2. 'Short' $i$-forms: forms and formations}

We present examples of symmetric $i$-pairs $(P ., \phi)$ in four cases $i=-1,0,1,2$ :
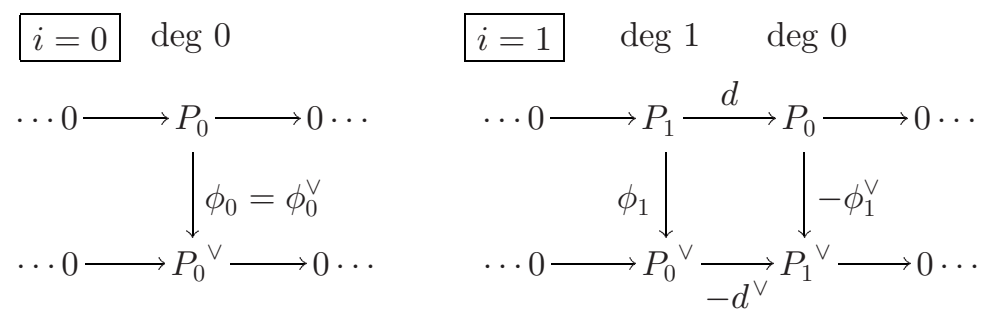

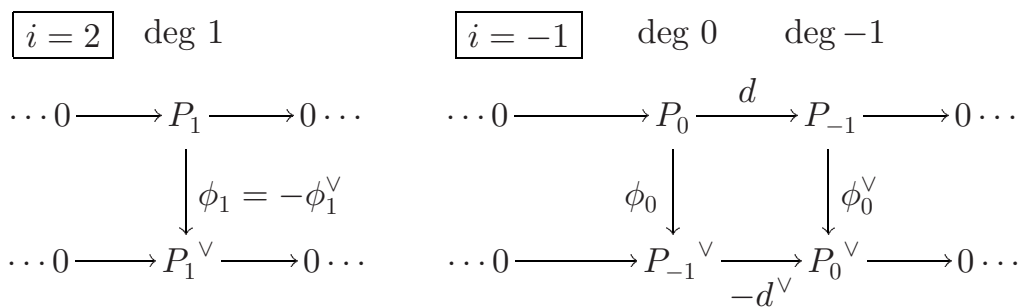

In each case, the complexes $P$. and $P^{\vee}$ are depicted horizontally and the symmetric $i$-form $\phi: P_{\bullet} \rightarrow \mathcal{D}_{X}\left(P_{\bullet}\right)[i]$ vertically. The symmetric pairs of the left-hand column are classical symmetric and skew-symmetric forms embedded in $\mathbb{D}^{\mathrm{b}}\left(\mathrm{VB}_{X}\right)$ via the functor $c_{0}$ (slightly pushed to the left for $i=2$ ). These symmetric $i$-pairs are $i$-spaces exactly when $\phi_{0}$ and $\phi_{1}$ are isomorphisms. The symmetric $i$-pairs of the right-hand column are $i$-spaces when $\phi$ is a quasi-isomorphism, that is, when its cone is an exact complex; these are formations; we call them symmetric if $i=-1$ and skew-symmetric if $i=1$. The four types of $i$-form presented above will be called short, for the obvious reasons.

\subsection{Product of symmetric spaces}

The precise definition of this product is given in [11], where the reader will also find an explanation for the existence of two different products - the left and the right one - which differ by signs. To fix the ideas, we will use here the left product. Let $\left(P_{\bullet}, \phi\right)$ be a symmetric $i$-form and $(Q, \psi)$ a symmetric $j$-form. The product

$$
(P ., \phi) \star(Q ., \psi)
$$

is then a symmetric $(i+j)$-form on the tensor product (of complexes) $P$. $\otimes_{\mathcal{O}_{X}} Q$. and we denote it by $\left(P_{\bullet} \otimes_{\mathcal{O}_{X}} Q_{\bullet}, \phi \star \psi\right)$. Up to signs and identifications like, for instance, $P . \otimes_{\mathcal{O}_{X}}\left(Q_{\bullet}^{\vee}[j]\right) \simeq\left(P_{\bullet} \otimes_{\mathcal{O}_{X}} Q_{\bullet}^{\vee}\right)[j]$, the morphism of complexes $\phi \star \psi$ is equal to the tensor product $\phi \otimes \psi$. Via $c_{0}$, this product coincides on short 0 -spaces with the usual tensor product of symmetric spaces as defined by Knebusch in [14].

\subsection{Symmetric cones}

We now recall the important cone construction. Let $\phi: P_{\bullet} \rightarrow \mathcal{D}_{X}\left(P_{\bullet}\right)[i]$ be a symmetric $i$-form (maybe not an isomorphism). Let $Q$. be the mapping cone of $\phi$. Then, there exists an isomorphism $\psi$ such that the following diagram commutes:

$$
\begin{aligned}
& P_{.} \longrightarrow \mathcal{D}_{X}\left(P_{\bullet}\right)[i] \stackrel{u}{\longrightarrow} Q . \stackrel{v}{\longrightarrow} P_{\bullet}[1] \\
& \left.\cong \downarrow(-1)^{i(i+1) / 2} \cdot \varpi_{P} \downarrow=\quad \psi \downarrow \simeq \quad(-1)^{i(i+1) / 2} \cdot \varpi_{P}[1]\right\rfloor \\
& \mathcal{D}_{X} \mathcal{D}_{X}\left(P_{\bullet}\right) \underset{\mathcal{D}_{X}(\phi)[i]}{\longrightarrow} \mathcal{D}_{X}(P \bullet)[i] \underset{-\mathcal{D}_{X}(v)[i+1]}{\longrightarrow} \mathcal{D}_{X}\left(Q_{\bullet}\right)[i+1] \underset{(-1)^{i} \mathcal{D}_{X}(u)[i+1]}{\longrightarrow} \mathcal{D}_{X} \mathcal{D}_{X}\left(P_{\bullet}\right)[1]
\end{aligned}
$$

If the isomorphism $\psi$ is moreover a symmetric $(i+1)$-form, we call such a diagram a cone diagram (over $\phi)$ and we say that $(Q ., \psi)$ is a symmetric cone of the pair $\left(P_{\bullet}, \phi\right)$.

Note that both rows of the diagram are exact triangles in $\mathbb{D}^{\mathrm{b}}\left(\mathrm{VB}_{X}\right)$ : the upper one by definition and the lower one is the dual of the upper row, shifted $i$ times. 
Assume for a moment that 2 is invertible over our scheme $X$. Then we can always choose the isomorphism $\psi$ to be a symmetric $(i+1)$-form; see [2]. Moreover, if $\left(Q_{.}^{\prime}, \psi^{\prime}\right)$ is another symmetric cone of $\phi$, then there exists an isometry $\left(Q_{\bullet}, \psi\right) \simeq\left(Q_{\bullet}^{\prime}, \psi^{\prime}\right)$. We say then that $(Q ., \psi)$ is the symmetric cone of $\phi$, in symbols:

$$
(Q ., \psi)=\operatorname{cone} \phi=\operatorname{cone}\left(P_{\bullet}, \phi\right) \text {. }
$$

\subsection{Witt groups}

The usual Witt group of symmetric (respectively skew-symmetric) spaces $\mathrm{W}_{\text {us }}(X)$ (respectively $\mathrm{W}_{\text {us }}^{-}(X)$ ) classifies these spaces up to isometry and modulo metabolic ones. More information about these Witt groups can be found in the fundamental paper of Knebusch [14]. The ith derived Witt group $\mathrm{W}^{i}(X)$ classifies symmetric $i$-spaces up to isometry and modulo neutral spaces, that is, spaces with Lagrangian (cf. $[\mathbf{2}, \S 2]$ ). In fact, a symmetric $i$-space is neutral exactly if it is a symmetric cone of some symmetric $(i-1)$-form, as described above. Observe that this definition does not require 2 to be invertible in $X$. We denote by $[P ., \phi]$ the Witt class of $(P ., \phi)$.

The Witt groups are contravariant functors. If $f: Y \rightarrow X$ is a morphism of schemes then the assignment $\left[P_{\bullet}, \phi\right] \mapsto\left[f^{*}\left(P_{\bullet}, \phi\right)\right]$ defines a homomorphism $f^{*}: \mathrm{W}^{i}(X) \rightarrow \mathrm{W}^{i}(Y)$ for all $i \in \mathbb{Z}$.

\subsection{Periodicity}

The derived Witt groups are 4-periodic. The shift by $2, P_{\bullet} \mapsto P .[2]$, induces an isomorphism $\tau: \mathrm{W}^{i}(X) \stackrel{\sim}{\rightarrow} \mathrm{W}^{i+4}(X)$ for all $i \in \mathbb{Z}$ and all schemes $X$. The same periodicity applies to the Witt groups with support defined below.

\subsection{Agreement}

We assume now that ' $X$ contains $\frac{1}{2}$ ', that is, that $X$ is a $\mathbb{Z}[1 / 2]$-scheme, that is, 2 is invertible in the ring $\Gamma\left(X, \mathcal{O}_{X}\right)$. The main result of $[\mathbf{3}]$ is that the functor $c_{0}: \mathrm{VB}_{X} \rightarrow \mathbb{D}^{\mathrm{b}}\left(\mathrm{VB}_{X}\right)$ induces isomorphisms:

$$
\mathrm{W}(X)=\mathrm{W}_{\mathrm{us}}(X) \stackrel{\simeq}{\longrightarrow} \mathrm{W}^{0}(X), \quad[P, \phi] \longmapsto\left[c_{0}(P), c_{0}(\phi)\right]
$$

and

$$
\mathrm{W}^{-}(X)=\mathrm{W}_{\mathrm{us}}^{-}(X) \stackrel{\simeq}{\longrightarrow} \mathrm{W}^{2}(X), \quad[Q, \psi] \longmapsto\left[c_{0}(Q)[1], c_{0}(\psi)[1]\right]
$$

\subsection{Localization (with $\frac{1}{2}$ )}

Other Witt groups appearing in this work are the Witt groups with support. For a complex $P_{\bullet} \in \mathbb{D}^{\mathrm{b}}\left(\mathrm{VB}_{X}\right)$ let

$$
\operatorname{supp} P_{\bullet}:=\left\{x \in X \mid H_{j}\left(P_{\bullet}\right)_{x} \neq 0 \text { for at least one } j\right\},
$$

be its (homological) support. Let $Z$ be a closed subscheme of $X$ with open complement $U$. The full triangulated subcategory of $\mathbb{D}^{\mathrm{b}}\left(\mathrm{VB}_{X}\right)$ which consists of complexes with support contained in $Z$ is denoted $\mathbb{D}_{Z}^{\mathrm{b}}\left(\mathrm{VB}_{X}\right)$. The restriction of the duality $\mathcal{D}_{X}$ to $\mathbb{D}_{Z}^{\mathrm{b}}\left(\mathrm{VB}_{X}\right)$ is again a duality, turning $\mathbb{D}_{Z}^{\mathrm{b}}\left(\mathrm{VB}_{X}\right)$ into a triangulated category with duality. The corresponding triangular Witt groups $\mathrm{W}_{Z}^{i}(X)(i \in \mathbb{Z})$ are called the derived Witt groups of $X$ with support in $Z$. They 
appear in the localization sequence of Balmer [2]. If $X$ is a regular scheme then there is an exact sequence

$$
\cdots \longrightarrow \mathrm{W}^{i}(X) \longrightarrow \mathrm{W}^{i}(U) \stackrel{\partial}{\longrightarrow} \mathrm{W}_{Z}^{i+1}(X) \longrightarrow \mathrm{W}^{i+1}(X) \longrightarrow \cdots .
$$

The connecting morphism $\partial$ comes from the cone construction (cf. $\S 3.4$ ) as follows. Let $w \in \mathrm{W}^{i}(U)$. Then $\partial(w)=\left[\operatorname{cone}\left(P_{\bullet}, \phi\right)\right]$, where $\left(P_{\bullet}, \phi\right)$ is any symmetric $i$-pair over $X$ with $\left[\left.(P ., \phi)\right|_{U}\right]=w$ (the existence of $(P ., \phi)$ is guaranteed by the regularity of $\left.X\right)$. The Witt groups with support are natural and so is the localization sequence.

\subsection{The graded Witt ring}

The (left) product of symmetric spaces of $\S 3.3$ yields a product structure

$$
\star: \mathrm{W}^{i}(X) \times \mathrm{W}_{Z}^{j}(X) \longrightarrow \mathrm{W}_{Z}^{i+j}(X), \quad\left(\left[P_{\bullet}, \phi\right],[Q ., \psi]\right) \longmapsto\left[\left(P_{\bullet}, \phi\right) \star(Q ., \psi)\right]
$$

for any $i, j \in \mathbb{Z}$, any scheme $X$ and closed subset $Z \subseteq X$. Via this pairing, $\mathrm{W}^{\mathrm{tot}}(X):=\bigoplus_{i \in \mathbb{Z}} \mathrm{W}^{i}(X)$ is a graded skew-commutative associative $\mathrm{W}^{0}(X)$-algebra, the graded Witt ring of $X$ and $\mathrm{W}_{Z}^{\text {tot }}(X):=\bigoplus_{i \in \mathbb{Z}} \mathrm{W}_{Z}^{i}(X)$ is a graded $\mathrm{W}^{\text {tot }}(X)$-module.

REMARK 3.3. Of course, Convention 2.2 applies here as well. For instance, if $X=\operatorname{Spec}(R)$ is affine and $Z \subset X$ is defined by the ideal $I$, we might say that a complex 'has support in the ideal $I$ ' and we shall write $\mathrm{W}_{I}^{i}(R)$ instead of $\mathrm{W}_{Z}^{i}(X)$.

\section{Basic facts about Koszul complexes}

In this section, $A$ is a ring, $\underline{T}=\left(T_{1}, \ldots, T_{n}\right)$ is any sequence in $A$, and $I:=\sum_{i=1}^{n} A T_{i}$ is the ideal generated by $\underline{T}$. As before, we write the dual as $M^{\vee}:=\operatorname{Hom}_{A}(M, A)$, for any $A$-module $M$.

We first recall the definition of the Koszul complex

$$
K_{\bullet}(A, \underline{T})=:\left(K_{\bullet}, d_{\bullet}\right) .
$$

Let $e_{1}, e_{2}, \ldots, e_{n}$ be a basis of the free $A$-module $A^{n}=\bigoplus_{i=1}^{n} A \cdot e_{i}$. The $A$-module $K_{i}=K_{i}(A, \underline{T}):=\bigwedge^{i} A^{n}$ is by definition the $i$ th exterior power of $A^{n}$. As is wellknown, the module $K_{i}$ is free with basis $\left\{e_{j_{1}} \wedge \ldots \wedge e_{j_{i}} \mid 1 \leqslant j_{1}<\ldots<j_{i} \leqslant n\right\}$. The differential $d_{i}=d_{i}(A, \underline{T}): K_{i} \rightarrow K_{i-1}$ is given by

$$
e_{j_{1}} \wedge \ldots \wedge e_{j_{i}} \longmapsto \sum_{k=1}^{i}(-1)^{k-1} T_{j_{k}} \cdot e_{j_{1}} \wedge \ldots \widehat{e_{j_{k}}} \ldots \wedge e_{j_{i}},
$$

where the symbol $\widehat{e_{j_{k}}}$ indicates that $e_{j_{k}}$ has been omitted. We consider this (homological) Koszul complex $K_{\bullet}(A, \underline{T})$ :

$$
\cdots 0 \longrightarrow K_{n}(A, \underline{T}) \stackrel{d_{n}(A, \underline{T})}{\longrightarrow} K_{n-1}(A, \underline{T}) \longrightarrow \cdots \stackrel{d_{1}(A, \underline{T})}{\longrightarrow} K_{0}(A, \underline{T}) \longrightarrow 0 \cdots
$$

as an element of $\mathbb{D}^{\mathrm{b}}\left(\mathrm{VB}_{A}\right)$ with $K_{j}(A, \underline{T})$ in degree $j$.

There is a structure of symmetric $n$-space on $K .(A, \underline{T})$ that we now give in an economic way; see more details in Remark 4.2. For each $i=1, \ldots, n$, let $K .\left(A, T_{i}\right) \in \mathbb{D}^{\mathrm{b}}\left(\mathrm{VB}_{A}\right)$ be the short Koszul complex for the one-element sequence 
$\left(T_{i}\right)$, that is,

$$
\begin{gathered}
K .\left(A, T_{i}\right)=\quad \cdots \longrightarrow 0 \longrightarrow \text { } A \stackrel{\cdot T_{i}}{\longrightarrow} A \longrightarrow 0 \longrightarrow \cdots \\
\operatorname{deg} 1 \quad \operatorname{deg} 0
\end{gathered}
$$

This complex can be equipped with the following symmetric 1-form:

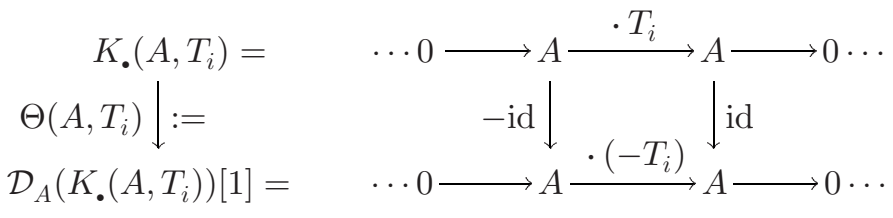

$$
\begin{aligned}
& \operatorname{deg} 1 \quad \operatorname{deg} 0
\end{aligned}
$$

where we identify $A=\operatorname{Hom}_{A}(A, A)$ as usual. This is the cone of the symmetric form $A \stackrel{\cdot\left(-T_{i}\right)}{\longrightarrow} A=\operatorname{Hom}_{A}(A, A)$, and so, in particular, a symmetric 1-space. It is easily checked that the tensor product of complexes $K_{\bullet}\left(A, T_{1}\right) \otimes_{A} \ldots \otimes_{A} K .\left(A, T_{n}\right)$ is equal to the Koszul complex $K_{\bullet}(A, \underline{T})$ of the sequence $\underline{T}=\left(T_{1}, \ldots, T_{n}\right)$ and therefore we can give the following definition.

Definition 4.1. With the above notation, we define a symmetric $n$-form

$$
\Theta(A, \underline{T}): K_{\bullet}(A, \underline{T}) \longrightarrow \mathcal{D}_{A}(K .(A, \underline{T}))[n]
$$

as the product (see $\S 3.3$ )

$$
\left(K_{\bullet}(A, \underline{T}), \Theta(A, \underline{T})\right):=\left(K \bullet\left(A, T_{1}\right), \Theta\left(A, T_{1}\right)\right) \star \ldots \star\left(K \bullet\left(A, T_{n}\right), \Theta\left(A, T_{n}\right)\right) .
$$

This defines a symmetric $n$-space $(K .(A, \underline{T}), \Theta(A, \underline{T}))$ which we call the canonical space on the Koszul complex $K .(A, \underline{T})$.

REMARK 4.2. To define this canonical space on $K .(A, \underline{T})$, it is not necessary to use the product structure of the derived Witt groups. The advantage of this approach is that we see at once that the canonical $n$-space is a symmetric $n$-space, but for calculations in the sequel it might be useful to have a good description of the symmetric $n$-form $\Theta(A, \underline{T})$. We define an isomorphism

$$
\rho: K_{\bullet}(A, \underline{T}) \stackrel{\simeq}{\longrightarrow} \mathcal{D}_{A}\left(K_{\bullet}(A, \underline{T})\right)[n]
$$

following $[7, \S 1.6]$. We fix for this an isomorphism $\omega: \bigwedge^{n}\left(A^{n}\right) \stackrel{\simeq}{\rightarrow} A$, and define an $A$-bilinear pairing

$$
b_{i}: K_{i}(A, \underline{T}) \times K_{n-i}(A, \underline{T}) \longrightarrow A
$$

by $(x, y) \mapsto \omega(x \wedge y)$ for all $0 \leqslant i \leqslant n$. This $b_{i}$ induces a homomorphism

$$
\varrho_{i}: K_{i}(A, \underline{T}) \longrightarrow \operatorname{Hom}_{A}\left(K_{n-i}(A, \underline{T}), A\right)=K_{n-i}(A, \underline{T})^{\vee}
$$

which is an isomorphism for all $0 \leqslant i \leqslant n$. It is straightforward to check (see [7, Proposition 1.6.10] if necessary) that

$$
d_{n-(i-1)}(A, \underline{T})^{\vee} \cdot \varrho_{i}=(-1)^{i-1} \varrho_{i-1} \cdot d_{i}(A, \underline{T}) .
$$

Consider the family of morphisms $\left(\rho_{i}\right)_{i \in \mathbb{Z}}$ defined by $\rho_{i}:=(-1)^{i(i+1) / 2+n(n-1) / 2} \cdot \varrho_{i}$ for $0 \leqslant i \leqslant n$, and $\rho_{i}:=0$ otherwise. This defines an isomorphism of complexes 
$\rho=\rho_{\bullet}: K_{\bullet}(A, \underline{T}) \rightarrow \mathcal{D}_{A}\left(K_{\bullet}(A, \underline{T})\right)[n]$, which coincides with the morphism of complexes $\Theta(A, \underline{T})$ as a thrilling calculation, using (in particular, the sign conventions of) [11, Example 1.4, Remark 1.9], shows.

By the following lemma this is easier to see if $\underline{T}$ is a regular sequence, which is the only interesting case for us here.

Lemma 4.3. Assume that $\underline{T}$ is a regular sequence. Identify $A \cong \operatorname{Hom}_{A}(A, A)$ as usual. Then for any morphism in $\mathbb{D}^{\mathrm{b}}\left(\mathrm{VB}_{A}\right)$ between the Koszul complex and its $n$-dual

$$
\varsigma: K_{\bullet}(A, \underline{T}) \longrightarrow \mathcal{D}_{A}\left(K_{\bullet}(A, \underline{T})\right)[n],
$$

there exists an $s \in A$ such that $\varsigma=s \cdot \Theta(A, \underline{T})$ in $\mathbb{D}^{\mathrm{b}}\left(\mathrm{VB}_{A}\right)$. If $s^{\prime} \in A$ is another element with this property then $s-s^{\prime} \in I$. The morphism $\varsigma$ is an isomorphism in $\mathbb{D}^{\mathrm{b}}\left(\mathrm{VB}_{A}\right)$ if and only if $s+I$ is a unit in the quotient ring $A / I$.

Proof. By assumption $\underline{T}=\left(T_{1}, \ldots, T_{n}\right)$ is a regular sequence and so the complex $K_{\bullet}(A, \underline{T})$ and its dual $\mathcal{D}_{A}\left(K_{\bullet}(A, \underline{T})\right)[n]$ are $A$-free resolutions of $A / I$ by [7, Proposition 1.6.10 and Corollary 1.6.14]. The lemma follows because $\Theta(A, \underline{T})$ is an isomorphism of complexes.

REMARK 4.4. It is clear that the restriction of $K_{\bullet}(A, \underline{T})$ becomes zero in the derived category $\mathbb{D}^{\mathrm{b}}\left(\mathrm{VB}_{A\left[T_{j}^{-1}\right]}\right)$ for all $1 \leqslant j \leqslant n$. Hence the complex $K_{\bullet}(A, \underline{T})$ has support in the closed subset of $\operatorname{Spec}(A)$ defined by the ideal $I=\sum_{j=1}^{n} A T_{j}$. Therefore the symmetric $n$-space $(K .(A, \underline{T}), \Theta(A, \underline{T}))$ defines an element in

$$
[K .(A, \underline{T}), \Theta(A, \underline{T})] \in \mathrm{W}_{I}^{n}(A) .
$$

Proposition 4.5. Let $1 \leqslant i \leqslant n$. Define the ideal $I_{i}:=\sum_{k \neq i} A T_{k}$ of $A$. Then $[K .(A, \underline{T}), \Theta(A, \underline{T})]=0$ in $\mathrm{W}_{I_{i}}^{n}(A)$.

Proof. The group $\mathrm{W}_{I_{i}}^{n-1}(A)$ obviously contains the element

$$
\begin{aligned}
& y:=\left[K_{\bullet}\left(A, T_{1}\right), \Theta\left(A, T_{1}\right)\right] \star \ldots \star\left[K .\left(A, T_{i-1}\right), \Theta\left(A, T_{i-1}\right)\right] \\
& \\
& \star \quad\left[K_{\bullet}\left(A, T_{i+1}\right), \Theta\left(A, T_{i+1}\right)\right] \star \ldots \star\left[K .\left(A, T_{n}\right), \Theta\left(A, T_{n}\right)\right] .
\end{aligned}
$$

Since the product is skew-commutative, we have

$$
\left[K_{\bullet}\left(A, T_{i}\right), \Theta\left(A, T_{i}\right)\right] \star y=(-1)^{i-1}\left[K_{\bullet}(A, \underline{T}), \Theta(A, \underline{T})\right],
$$

where we consider $\left[K .\left(A, T_{i}\right), \Theta\left(A, T_{i}\right)\right]$ as an element of $\mathrm{W}^{1}(A)$. Therefore the result follows from the observation that this element is indeed zero in $\mathrm{W}^{1}(A)$. In fact, the complex $c_{0}(A) \in \mathbb{D}^{\mathrm{b}}\left(\mathrm{VB}_{A}\right)$ is a Lagrangian (cf. $\left.[\mathbf{2}, \S 2]\right)$ of the symmetric 1-space $\left(K_{\bullet}\left(A, T_{i}\right), \Theta\left(A, T_{i}\right)\right)$ :
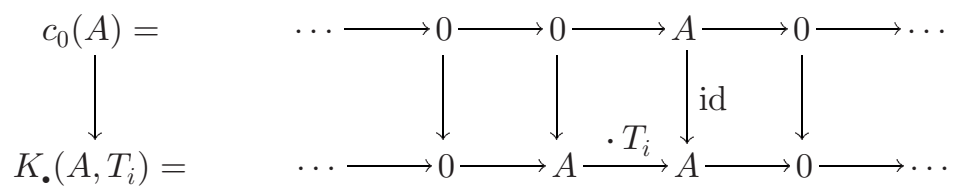

and so $\left[K_{\bullet}\left(A, T_{i}\right), \Theta\left(A, T_{i}\right)\right]=0$ in $\mathrm{W}^{1}(A)$. 
In the above proof, note that the class $y \in \mathrm{W}_{I_{i}}^{n-1}(A)$ does not belong to $\mathrm{W}_{I}^{n-1}(A)$ and thus the argument cannot be used to deduce that $[K .(A, \underline{T}), \Theta(A, \underline{T})]=0$ in $\mathrm{W}_{I}^{n}(A)$. On the contrary, see Theorem 9.2.

Corollary 4.6. We have $\left[K_{\bullet}(A, \underline{T}), \Theta(A, \underline{T})\right]=0$ in $\mathrm{W}^{n}(A)$.

Proof. This is clear since $\mathrm{W}_{I}^{n}(A) \rightarrow \mathrm{W}^{n}(A)$ factors via $\mathrm{W}_{I_{1}}^{n}(A)$, for instance.

REMARK 4.7. Our 'canonical' Koszul symmetric space $[K .(A, \underline{T}), \Theta(A, \underline{T})]$ is only canonical up to sign. In fact, its definition obviously depends on sign conventions, as usual when working in derived categories, as well as some personal sign choices as, for example, in the definition of the symmetric 1-form on the Koszul complex of length 1 , at the beginning of this section. The reader should not consider this sign question as crucial and can equally well use his own set of conventions. Applying Lemma 4.3 to $A=\mathbb{Z}\left[T_{1}, \ldots, T_{n}\right]$ and to $\underline{T}=\left(T_{1}, \ldots, T_{n}\right)$, as we shall do below, we get $A / I=\mathbb{Z}$ and no other unit than \pm 1 can really enter the game.

Indeed, our main result Theorem 9.13 says that for any regular scheme $X$ of finite Krull dimension the total Witt ring $\mathrm{W}^{\text {tot }}\left(\mathbb{U}_{X}^{n}\right)$ is a graded $\mathrm{W}^{\text {tot }}(X)$-algebra with only one generator $\varepsilon$ which satisfies the relation $\varepsilon^{2}=0$ if $n \geqslant 2$ and $\varepsilon^{2}=1$ if $n=1$, so, this result clearly does not depend on any choice yielding a sign change in the definition of $\varepsilon$. A reader who prefers different sign conventions would maybe get from our construction $-\varepsilon$ as generator which obviously satisfies the same relation as $\varepsilon$. Once again, these signs are not really relevant and we could have written everything 'up to sign' using Lemma 4.3 extensively. We only carry the (hopefully) exact signs throughout the paper for sake of consistency.

\section{Koszul cut in two}

We want to 'split' the Koszul complex of $\S 4$ into two pieces, dual to each other. This is easy to understand but a little tricky to write. Recall our running conventions of $\S 2$ that $r+4 q=n-1$ (see (1)) and that $\ell:=\left[\frac{n}{2}\right]$ (see (2)). Now, more precisely, we want to define a symmetric $r$-pair $\left(C_{\bullet}(A, \underline{T}), \Xi(A, \underline{T})\right)$, such that there is an isometry

$$
\operatorname{cone}(C \cdot(A, \underline{T}), \Xi(A, \underline{T})) \simeq\left(K_{\bullet}(A, \underline{T}), \Theta(A, \underline{T})\right)[-2 q] .
$$

We abbreviate the canonical form on $K_{\bullet}:=K_{\bullet}(A, \underline{T})$ by $\Theta:=\Theta(A, \underline{T})$, and set

$$
S=S(A, \underline{T}):=\operatorname{Coker}\left(K_{\ell+2}(A, \underline{T}) \stackrel{d_{\ell+2}(A, \underline{T})}{\longrightarrow} K_{\ell+1}(A, \underline{T})\right) .
$$

Let $\operatorname{pr}_{S}=\operatorname{pr}_{S(A, \underline{T})}: K_{\ell+1} \rightarrow S=$ Coker $d_{\underline{\ell+2}}$ be the projection. Since $d_{\ell+1} d_{\ell+2}=0$, there exists a unique morphism $\bar{d}_{\ell+1}=\bar{d}_{\ell+1}(A, \underline{T}): S \rightarrow K_{\ell}$, such that

$$
d_{\ell+1}(A, \underline{T})=\bar{d}_{\ell+1}(A, \underline{T}) \cdot \operatorname{pr}_{S} .
$$

For each $j=0, \ldots, n$, we have $\operatorname{rank}_{A}\left(K_{j}\right)=\left(\begin{array}{c}n \\ j\end{array}\right)$. In particular, if $n=2 \ell+1$ is odd, we have $\operatorname{rank}_{A} K_{\ell}=\operatorname{rank}_{A} K_{\ell+1}$ and life will be easy. When $n=2 \ell$ is even, $K_{\ell}$ has maximal (even) rank $\left(\begin{array}{c}2 \ell \\ \ell\end{array}\right)$ and we need some preparatory considerations. In this case, the symmetric $n$-form $\Theta_{\bullet}: K_{\bullet} \stackrel{\cong}{\rightarrow} \mathcal{D}_{A}\left(K_{\bullet}\right)[n]$ gives an isomorphism

$$
\Theta_{\ell}: K_{\ell} \longrightarrow K_{\ell}^{\vee}=\operatorname{Hom}_{A}\left(K_{\ell}, A\right)
$$

which is symmetric if $\ell$ is even and skew-symmetric otherwise. 
Lemma 5.1. If $n=2 \ell$ is even, there exist two totally isotropic subspaces $L$ and $M$ of $\left(K_{\ell}, \Theta_{\ell}\right)$, of the same rank $\frac{1}{2}\left(\begin{array}{c}2 \ell \\ \ell\end{array}\right)$, such that $K_{\ell}=L \oplus M$ and such that $\Theta_{\ell}$ becomes

$$
\Theta_{\ell}=\left(\begin{array}{cc}
0 & (-1)^{\ell} \lambda^{\vee} \operatorname{can}_{M} \\
\lambda & 0
\end{array}\right): K_{\ell}=L \oplus M \longrightarrow L^{\vee} \oplus M^{\vee}=K_{\ell}^{\vee}
$$

where $\lambda: L \stackrel{\simeq}{\rightarrow} M^{\vee}$ is an isomorphism. Moreover, we have

$$
(-1)^{\ell} d_{\ell+1}^{\vee} \cdot\left(\operatorname{pr}_{L}\right)^{\vee} \cdot \lambda^{\vee} \cdot \operatorname{can}_{M} \cdot \operatorname{pr}_{M} \cdot d_{\ell+1}+d_{\ell+1}^{\vee} \cdot\left(\operatorname{pr}_{M}\right)^{\vee} \cdot \lambda \cdot \operatorname{pr}_{L} \cdot d_{\ell+1}=0,
$$

where $\operatorname{pr}_{L}: K_{\ell} \rightarrow L$ and $\operatorname{pr}_{M}: K_{\ell} \rightarrow M$ denote the projections.

Proof. Let $e_{1}, \ldots, e_{n}$ be a basis of $A^{n}$ and define $L:=e_{1} \wedge \wedge^{\ell-1} A^{n}$. The space

$$
M:=\bigoplus_{2 \leqslant i_{1}<i_{2}<\ldots<i_{\ell} \leqslant n} A \cdot e_{i_{1}} \wedge e_{i_{2}} \wedge \ldots \wedge e_{i_{\ell}}
$$

is obviously a complement of $L$ in $K_{\ell}=\bigwedge^{\ell} A^{n}$ and both subspaces have rank $\left(\begin{array}{c}2 \ell-1 \\ \ell-1\end{array}\right)=\left(\begin{array}{c}2 \ell-1 \\ \ell\end{array}\right)=\frac{1}{2}\left(\begin{array}{c}2 \ell \\ \ell\end{array}\right)$. Now use the description of $\Theta_{\ell}$ given in Remark 4.2. Let $\omega: \bigwedge^{n} A^{n} \cong A$ be the isomorphism which sends $e_{1} \wedge \ldots \wedge e_{n}$ to $1 \in A$. Then $\Theta_{\ell}(x)(y)= \pm \omega(x \wedge y)$. From this we easily see that both subspaces are totally isotropic: for $L$ it is because $e_{1} \wedge e_{1}=0$ and for $M$ it is because two subsets with $\ell$ elements in $\{2, \ldots, n\}$ must intersect. Since $\Theta_{\ell}$ is a $(-1)^{\ell}$-symmetric isomorphism, its decomposition in $L \oplus M$ must be as claimed in the lemma. Equation (6) follows from the fact that $\Theta: K_{\bullet} \rightarrow \mathcal{D}_{A}\left(K_{\bullet}\right)[n]$ is a morphism of complexes.

\subsection{Definition of $(C .(A, \underline{T}), \Xi(A, \underline{T}))$}

As the above discussion shows, we will have to distinguish the cases where $n$ is odd from those where $n$ is even and the definition extends over Cases 5.1.1-5.1.4 below. We shall consider a sign $\epsilon_{n} \in\{-1,1\}$ which will be fixed later on; see $\S 7.1$.

We start with $n=2 \ell+1$ odd.

5.1.1. Case $r=0$. Here $\ell=2 q$ is even and $(C \cdot(A, \underline{T}), \Xi(A, \underline{T}))$ is defined to be the following symmetric 0-pair:

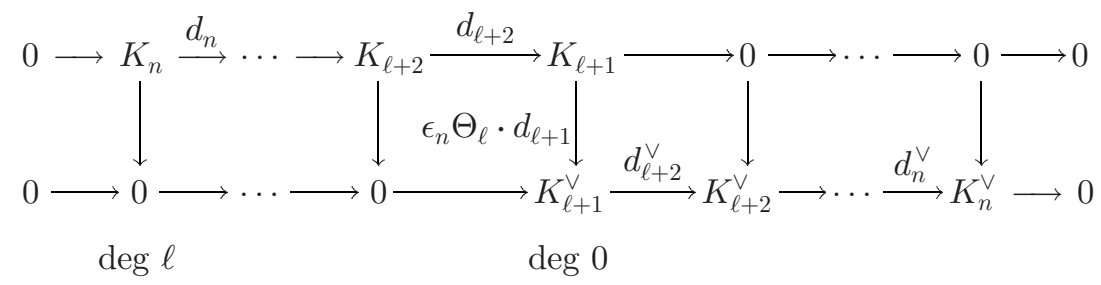

If $\underline{T}$ is a regular sequence then the Koszul complex $K$. is exact and so we have the following quasi-isomorphism:

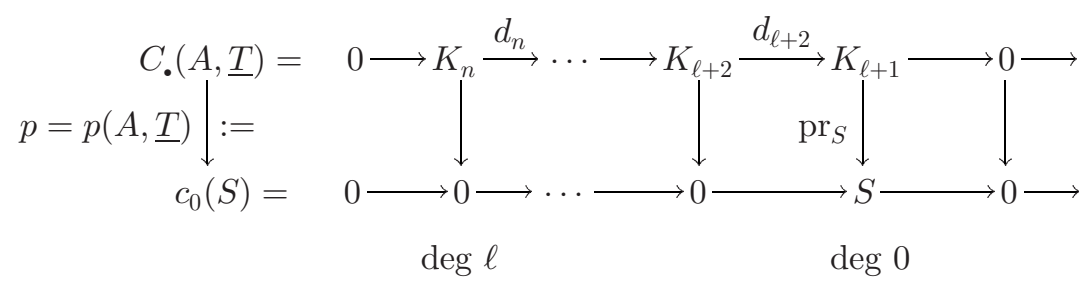


5.1.2. Case $r=2$. Here $\ell=2 q+1$ is odd and $(C .(A, \underline{T}), \Xi(A, \underline{T}))$ is defined to be the following symmetric 2-pair:

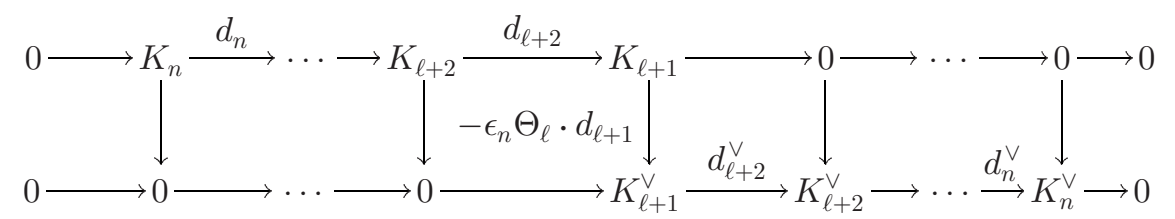

$\operatorname{deg} \ell+1$

$\operatorname{deg} 1$

As above, if $\underline{T}$ is a regular sequence, the projection $\operatorname{pr}_{S}: K_{\ell+1} \rightarrow S$ induces a quasi-isomorphism of complexes

$$
p=p(A, \underline{T}): C_{\bullet}(A, \underline{T}) \longrightarrow c_{0}(S)[1] .
$$

Now let $n=2 \ell$ be even.

We fix two totally isotropic subspaces $L$ and $M$ of $K_{\ell}$ and an isomorphism $\lambda: L \rightarrow M^{\vee}$ as in Lemma 5.1 and keep notation as there. We set

$$
h:=\lambda^{\vee} \cdot \operatorname{can}_{M} \cdot \operatorname{pr}_{M} \cdot d_{\ell+1}: K_{\ell+1} \longrightarrow L^{\vee}
$$

We now define the space $(C \cdot(A, \underline{T}), \Xi(A, \underline{T}))$ for $n$ even. It follows from equation (6) in Lemma 5.1 that both squares in the middle of the two diagrams below commute and so the morphism $\Xi(A, \underline{T})$ is really a morphism of complexes.

5.1.3. Case $r=-1$. Here $\ell=2 q$ is even and $(C \cdot(A, \underline{T}), \Xi(A, \underline{T}))$ is defined to be the following symmetric $(-1)$-pair:

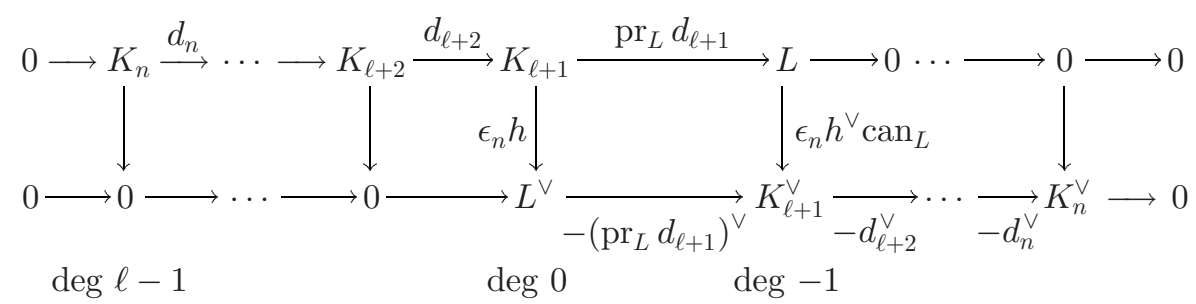

If the sequence $\underline{T}$ is regular, the homology of $C \cdot(A, \underline{T})$ is not concentrated in one degree (as in the case $n$ odd) but there exists a 'short' complex $F_{0}(A, \underline{T})$ defined as follows and which is quasi-isomorphic to $C .(A, \underline{T})$ :

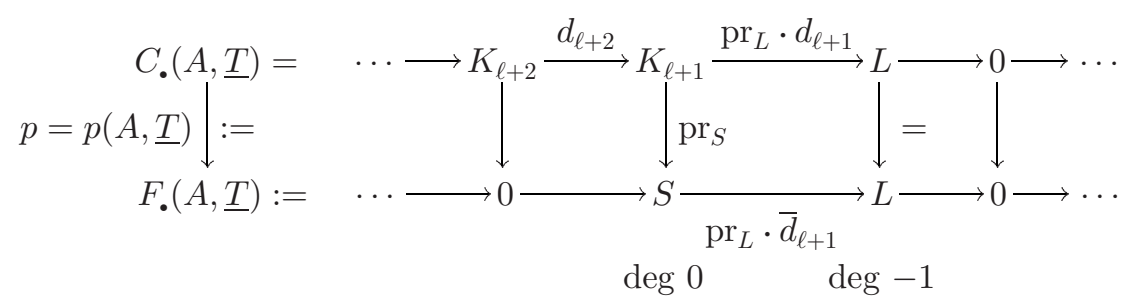


5.1.4. Case $r=1$. Here $\ell=2 q+1$ is odd and $(C .(A, \underline{T}), \Xi(A, \underline{T}))$ is defined to be the following symmetric 1-pair:

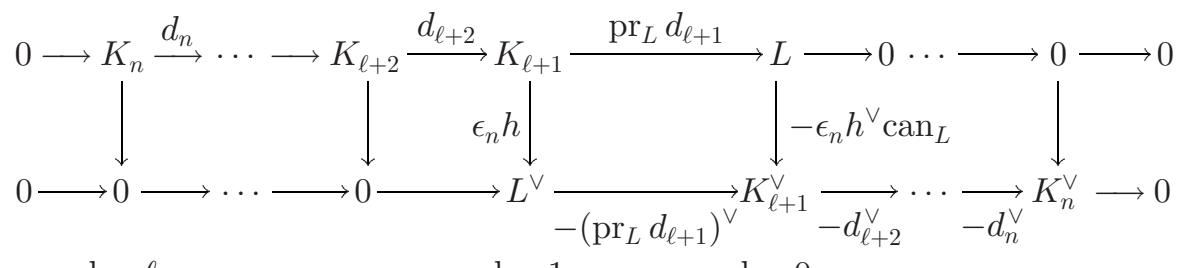

$\operatorname{deg} \ell$

$\operatorname{deg} 1$

$\operatorname{deg} 0$

As in the case $r=-1$, when $T$ is a regular sequence, we have a quasi-isomorphism $p=p(A, \underline{T}): C_{\bullet}(A, \underline{T}) \rightarrow F_{\bullet}(\bar{A}, \underline{T})$, where $F_{\bullet}(A, \underline{T})$ is now the complex

$$
\cdots \longrightarrow 0 \longrightarrow \text { } S \stackrel{\operatorname{pr}_{L} \cdot \bar{d}_{\ell+1}}{\longrightarrow} L \longrightarrow 0 \longrightarrow \cdots
$$

REMARK 5.2. Let $f: A \rightarrow B$ be a morphism of rings. The natural isomorphism

$$
f^{*}\left(K_{\bullet}(A, \underline{T})\right)=K_{\bullet}(A, \underline{T}) \otimes_{A} B \stackrel{\simeq}{\longrightarrow} K_{\bullet}(B, f(\underline{T}))
$$

induces a natural isometry

$$
f^{*}\left(K_{\bullet}(A, \underline{T}), \Theta(A, \underline{T})\right) \simeq(K .(B, f(\underline{T})), \Theta(B, f(\underline{T}))) .
$$

Restricting this isomorphism above to the subcomplex $C .(A, \underline{T})$ we get a natural isometry $f^{*}(C \cdot(A, \underline{T}), \Xi(A, \underline{T})) \simeq(C \cdot(B, f(\underline{T})), \Xi(B, f(\underline{T})))$.

LEMMA 5.3. The mapping cone of the morphism $\Xi(A, \underline{T})$ is isomorphic (as a complex) to $K_{.}(A, \underline{T})[-2 q]$.

Proof. This is an easy direct computation, which we leave to the reader. It is clear in the cases where $n$ is odd and it requires Lemma 5.1 for $n$ even. In all four cases, we use the isomorphism $\Theta$ to replace the $K_{j}^{\vee}$ by $K_{n-j}$ for $j \geqslant \ell+1$.

\section{The Koszul symmetric space $\mathbf{K}_{X}^{(n)}$ over $\mathbb{A}_{X}^{n}$}

Let $R$ be a ring. We apply the constructions of $\S 4$ to $A:=R\left[T_{1}, \ldots, T_{n}\right]$, the polynomial ring in $n$ variables over $R$, and to the sequence $\underline{T}:=\left(T_{1}, \ldots, T_{n}\right)$. The reader can think of $R=\mathbb{Z}$ or $R=\mathbb{Z}[1 / 2]$, since these are the important cases, from which the rest will be deduced.

Definition 6.1. The Koszul symmetric $n$-space $\mathbf{K}_{R}^{(n)}=\left(\mathrm{K}_{R}^{(n)}, \Theta_{R}^{(n)}\right)$ over $\mathbb{A}_{R}^{n}$ is the symmetric $n$-space where $K_{R}^{(n)}:=K \cdot(A, \underline{T})$ is the Koszul complex over $\mathbb{A}_{R}^{n}$ and the symmetric $n$-form $\Theta_{R}^{(n)}:=\Theta(A, \underline{T})$ is the one of Definition 4.1.

REMARK 6.2. Pay attention: $\mathbf{K}_{R}^{(n)}$ is a symmetric $n$-space defined over the ring $A=R\left[T_{1}, \ldots, T_{n}\right]$ and not over the ring $R$, as the notation might suggest. 
It is clear that the Koszul symmetric $n$-space behaves well with respect to basechange. More precisely, let $f: R \rightarrow R^{\prime}$ be a morphism of rings and let

$$
\alpha_{f}: R\left[T_{1}, \ldots, T_{n}\right] \longrightarrow R^{\prime}\left[T_{1}, \ldots, T_{n}\right]
$$

be the obvious induced morphism. Then, by Remark 5.2 there is a natural isometry

$$
\alpha_{f}^{*}\left(\mathbf{K}_{R}^{(n)}\right) \stackrel{\simeq}{\longrightarrow} \mathbf{K}_{R^{\prime}}^{(n)}
$$

In particular, $\mathbf{K}_{R}^{(n)}$ is extended from $\mathbf{K}_{\mathbb{Z}}^{(n)}$. This justifies the following extension of Definition 6.1 .

Definition 6.3. Let $X$ be a scheme. We define the symmetric $n$-space

$$
\mathbf{K}_{X}^{(n)}:=\alpha_{X}^{*}\left(\mathbf{K}_{\mathbb{Z}}^{(n)}\right)
$$

where $\alpha_{X}: \mathbb{A}_{X}^{n} \rightarrow \mathbb{A}_{\mathbb{Z}}^{n}$ is the base-change morphism; see (3). We call $\mathbf{K}_{X}^{(n)}$ the Koszul symmetric $n$-space over $\mathbb{A}_{X}^{n}$. As before, we denote the underlying complex of free $\mathcal{O}_{\mathbb{A}_{X}^{n}}$-modules and its symmetric $n$-form by

$$
\mathrm{K}_{X}^{(n)}=\alpha_{X}^{*}\left(\mathrm{~K}_{\mathbb{Z}}^{(n)}\right) \quad \text { and } \quad \Theta_{X}^{(n)}=\alpha_{X}^{*}\left(\Theta_{\mathbb{Z}}^{(n)}\right) .
$$

REMARK 6.4. It is obvious from the definition that for any morphism of schemes $f: Y \rightarrow X$ we have an isometry $\alpha_{f}^{*}\left(\mathbf{K}_{X}^{(n)}\right) \simeq \mathbf{K}_{Y}^{(n)}$ over $\mathbb{A}_{Y}^{n}$.

Definition 6.5. By Remark 4.4, the complex $K_{X}^{(n)}$ has support in the closed subset $\mathbb{A}_{X}^{n} \backslash \mathbb{U}_{X}^{n}$ of $\mathbb{A}_{X}^{n}$ which we identify with $X$ in the following via the zero section of the bundle $\mathbb{A}_{X}^{n} \rightarrow X$. Therefore, the symmetric $n$-space $\mathbf{K}_{X}^{(n)}$ represents a Witt class

$$
\kappa_{X}^{(n)}:=\left[\mathbf{K}_{X}^{(n)}\right] \quad \in \mathrm{W}_{\mathbb{A}_{X}^{n} \backslash \mathbb{U}_{X}^{n}}^{n}\left(\mathbb{A}_{X}^{n}\right)=\mathrm{W}_{X}^{n}\left(\mathbb{A}_{X}^{n}\right) .
$$

\section{The half-Koszul symmetric space $\mathbf{E}_{X}^{(n)}$ over $\mathbb{U}_{X}^{n}$}

Definition 7.1. Let $R$ be a ring. We now apply the splitting of $\S 5$ to the space $\mathbf{K}_{R}^{(n)}$ of $\S 6$. As above, we put $A:=R\left[T_{1}, \ldots, T_{n}\right]$ and $\underline{T}:=\left(T_{1}, \ldots, T_{n}\right)$. We define

$$
\mathrm{C}_{R}^{(n)}:=C_{\bullet}(A, \underline{T}) \quad \text { and } \quad \Xi_{R}^{(n)}:=\Xi(A, \underline{T})
$$

as defined in 5.1.1 to 5.1.4. For any scheme $X$ we define

$$
\mathrm{C}_{X}^{(n)}:=\alpha_{X}^{*}\left(\mathrm{C}_{\mathbb{Z}}^{(n)}\right) \quad \text { and } \quad \Xi_{X}^{(n)}:=\alpha_{X}^{*}\left(\Xi_{\mathbb{Z}}^{(n)}\right)
$$

where $\alpha_{X}: \mathbb{A}_{X}^{n} \rightarrow \mathbb{A}_{\mathbb{Z}}^{n}$ is the base-change morphism. This coincides with the above in the affine case by Remark 5.2. For all $n \in \mathbb{N}$, the symmetric $r$-pair $\left(\mathrm{C}_{X}^{(n)}, \Xi_{X}^{(n)}\right)$ on $\mathbb{A}_{X}^{n}$ will be denoted by $\mathbf{C}_{X}^{(n)}$.

\subsection{The symmetric cone of $\mathbf{C}_{X}^{(n)}$}

Instead of calculating cone $\left(\mathbf{C}_{X}^{(n)}\right)$ directly (which is possible, but cumbersome) we take full advantage of Lemma 4.3. More precisely, we use the fact that any quasiisomorphism $K_{\mathbb{Z}}^{(n)} \rightarrow \mathcal{D}_{\mathbb{Z}\left[T_{1}, \ldots, T_{n}\right]}\left(K_{\mathbb{Z}}^{(n)}\right)[n]$ is equal to the symmetric $n$-form $\pm \Theta_{\mathbb{Z}}^{(n)}$ in $\mathbb{D}^{\mathrm{b}}\left(\mathrm{VB}_{\mathbb{Z}\left[T_{1}, \ldots, T_{n}\right]}\right)$.

So let, for a moment, $R=\mathbb{Z}$ and $A=\mathbb{Z}\left[T_{1}, \ldots, T_{n}\right]$. We abbreviate $K_{\bullet}:=K_{\bullet}(A, \underline{T})$ and $\Theta=\Theta(A, \underline{T})$. We get from Lemma 5.3 the following commutative diagram 
$\left(\right.$ where $\left.\mathcal{D}=\mathcal{D}_{A}\right)$ :

$$
\begin{aligned}
& \mathrm{C}_{\mathbb{Z}}^{(n)} \stackrel{\Xi_{\mathbb{Z}}^{(n)}}{\longrightarrow} \mathcal{D}\left(\mathrm{C}_{\mathbb{Z}}^{(n)}\right)[r] \stackrel{u}{\longrightarrow} K \cdot[-2 q] \longrightarrow \mathrm{C}_{\mathbb{Z}}^{(n)}[1] \\
& \left|(-1)^{r(r+1) / 2} \varpi_{\mathrm{C}_{\mathbb{Z}}^{(n)}}\right|=\quad(-1)^{r(r+1) / 2} \varpi_{\mathrm{C}_{\mathbb{Z}}^{(n)}}[1] \downarrow \\
& \mathcal{D} \mathcal{D}\left(\mathrm{C}_{\mathbb{Z}}^{(n)}\right) \underset{\mathcal{D}\left(\Xi_{\mathbb{Z}}^{(n)}\right)[r]}{\longrightarrow} \mathcal{D}\left(\mathrm{C}_{\mathbb{Z}}^{(n)}\right)[r] \underset{-\mathcal{D}(v)[r+1]}{\longrightarrow} \mathcal{D}\left(K_{\bullet}[-2 q]\right)[r+1] \underset{(-1)^{r} \mathcal{D}(u)[r+1]}{\longrightarrow} \mathcal{D} \mathcal{D}\left(\mathrm{C}_{\mathbb{Z}}^{(n)}\right)[1]
\end{aligned}
$$

Here the rows are exact triangles for all $n \in \mathbb{N}$ (the bottom row is the dual of the upper one, shifted $r$ times). By the very basic properties of triangulated categories there exists an isomorphism

$$
\varsigma: K_{\bullet}[-2 q] \longrightarrow \mathcal{D}\left(K_{\bullet}[-2 q]\right)[r+1]=\left(\mathcal{D}\left(K_{\bullet}\right)[n]\right)[-2 q],
$$

in $\mathbb{D}^{\mathrm{b}}\left(\mathrm{VB}_{A}\right)$ such that diagram $(7)$ commutes. By Lemma 4.3 the isomorphism $\varsigma$ is equal to $\pm \Theta[-2 q]$. Replacing $\Xi_{\mathbb{Z}}^{(n)}$ by $-\Xi_{\mathbb{Z}}^{(n)}$ if necessary, that is, replacing $\epsilon_{n}$ by $-\epsilon_{n}$ in the definition of $\mathbf{C}_{R}^{(n)}$, we can assume that $\varsigma=\Theta[-2 q]$ for all $n \in \mathbb{N}$, that is, $(K \cdot[-2 q], \varsigma)=\mathbf{K}_{\mathbb{Z}}^{(n)}[-2 q]$ for all $n \in \mathbb{N}$.

We fix $\epsilon_{n}$ as explained above, namely $\epsilon_{n}$ is the unique element in $\{-1,1\}$, such that cone $\left(\mathbf{C}_{\mathbb{Z}}^{(n)}\right)=\mathbf{K}_{\mathbb{Z}}^{(n)}[-2 q]$.

REMARK 7.2. A straightforward but slightly cumbersome calculation shows that

$$
\epsilon_{n}=(-1)^{n(n+1) / 2}
$$

Note that this depends on the sign choices made in 5.1.1-5.1.4. The latter have been made such that formula (8) is true.

However, as already said in Remark 4.7, these signs are not important for our work. We only need the fact that there exists a symmetric $r$-pair $\mathbf{C}_{\mathbb{Z}}^{(n)}$ over $\mathbb{A}_{\mathbb{Z}}^{n}$, such that cone $\mathbf{C}_{\mathbb{Z}}^{(n)}=\mathbf{K}_{\mathbb{Z}}^{(n)}[-2 q]$.

We can now calculate cone $\left(\mathbf{C}_{X}^{(n)}\right)$ for any scheme $X$ and any $n \in \mathbb{N}$. The pullback via the base-change morphism $\alpha_{X}: \mathbb{A}_{X}^{n} \rightarrow \mathbb{A}_{\mathbb{Z}}^{n}$ of diagram (7) above is a cone diagram for the symmetric $r$-form $\alpha_{X}^{*}\left(\mathbf{C}_{\mathbb{Z}}^{(n)}\right)$. We have an isometry $\mathbf{C}_{X}^{(n)} \simeq \alpha_{X}^{*}\left(\mathbf{C}_{\mathbb{Z}}^{(n)}\right)$ (cf. Remark 5.2) and so we get $\operatorname{cone}\left(\mathbf{C}_{X}^{(n)}\right) \simeq \alpha_{X}^{*}\left(\mathbf{K}_{\mathbb{Z}}^{(n)}[-2 q]\right) \simeq \alpha_{X}^{*}\left(\mathbf{K}_{\mathbb{Z}}^{(n)}\right)[-2 q]$ (cf. Lemma B.1 for the later isometry). We have proven the following result.

TheOrem 7.3. With this choice of $\epsilon_{n}$, the cone of the symmetric pair $\mathbf{C}_{X}^{(n)}$ is the Koszul symmetric space shifted as follows:

$$
\operatorname{cone}\left(\mathbf{C}_{X}^{(n)}\right)=\mathbf{K}_{X}^{(n)}[-2 q] \text {. }
$$

In particular, $\left.\Xi_{X}^{(n)}\right|_{\mathbb{U}_{X}^{n}}$ is an isomorphism in $\mathbb{D}^{\mathrm{b}}\left(\mathrm{VB}_{\mathbb{U}_{X}^{n}}\right)$ because the homology of $\left.K \cdot\right|_{X} ^{n}$ vanishes.

Definition 7.4. Let $X$ be a scheme. The symmetric $r$-space

$$
\mathbf{E}_{X}^{(n)}:=\left.\mathbf{C}_{X}^{(n)}\right|_{\mathbb{U}_{X}^{n}}
$$

will be called the half-Koszul space over the scheme $X$. Its Witt class is denoted by

$$
\varepsilon_{X}^{(n)}:=\left[\mathbf{E}_{X}^{(n)}\right] \quad \in \mathrm{W}^{r}\left(\mathbb{U}_{X}^{n}\right) .
$$

The following result is obvious (cf. Remark 5.2). 
Lemma 7.5. Let $f: Y \rightarrow X$ be a morphism of schemes. Then there is a natural isometry

$$
v_{f}^{*}\left(\varepsilon_{X}^{(n)}\right) \stackrel{\simeq}{\longrightarrow} \varepsilon_{Y}^{(n)}
$$

8. The short symmetric space $\mathbf{F}_{X}^{(n)}$ over $\mathbb{U}_{X}^{n}$

By the main result of $[\mathbf{3}]$ we know that $\mathbf{E}_{X}^{(n)}$ is Witt equivalent to a space living on a short complex; see $\S 3.2$. In fact, we will now see that $\mathbf{E}_{X}^{(n)}$ is not only Witt equivalent, but isometric to such a 'short symmetric space'.

We use the notation of 5.1.1-5.1.4 with $R=\mathbb{Z}$, that is, $A=\mathbb{Z}\left[T_{1}, \ldots, T_{n}\right]$ is the polynomial ring in $n$ variables over $\mathbb{Z}, \underline{T}=\left(T_{1}, \ldots, T_{n}\right)$, and $K_{\bullet}=K_{\bullet}(A, \underline{T})$ is the Koszul complex of the sequence $\underline{T}$ over $A$. As in 5.1.1-5.1.4 we denote the differential of this Koszul complex by $d_{\bullet}$ and set

$$
S=S(A, \underline{T})=\text { Coker }\left(K_{\ell+2} \stackrel{d_{\ell+2}}{\longrightarrow} K_{\ell+1}\right) .
$$

Note that $\underline{T}$ is a regular sequence and so $K$. is a finite free resolution of $\mathbb{Z} \simeq A / I$, where $I$ is the ideal generated by $\underline{T}$. It follows that $K .\left.(A, \underline{T})\right|_{\operatorname{Spec} A\left[T_{i}^{-1}\right]}$ is a split exact sequence and so

$$
\mathcal{E}_{\mathbb{Z}}^{(n)}:=\left.\operatorname{Coker}\left(K_{\ell+2} \stackrel{d_{\ell+2}}{\longrightarrow} K_{\ell+1}\right)\right|_{\mathbb{U}_{\mathbb{Z}}^{n}}=\left.\left.S\right|_{\mathbb{U}_{\mathbb{Z}}^{n}} \simeq \operatorname{Ker} d_{\ell}\right|_{\mathbb{U}_{\mathbb{Z}}^{n}}
$$

is a locally free $\mathcal{O}_{\mathbb{U}_{\mathbb{Z}}^{n}}$-module of rank $\sum_{i=0}^{\ell}(-1)^{i}\left(\begin{array}{c}n \\ \ell-i\end{array}\right)=\left(\begin{array}{c}n-1 \\ n-\ell-1\end{array}\right)$. Clearly the same is true for the pull-back

$$
\mathcal{E}_{X}^{(n)}:=v_{X}^{*}\left(\mathcal{E}_{\mathbb{Z}}^{(n)}\right),
$$

where $v_{X}: \mathbb{U}_{X}^{n} \rightarrow \mathbb{U}_{\mathbb{Z}}^{n}$ is induced by base change; see (3). Note that we have

$$
\mathcal{E}_{X}^{(n)}=\left.\left.\operatorname{Coker}\left(\mathrm{K}_{X, \ell+2}^{(n)} \longrightarrow \mathrm{K}_{X, \ell+1}^{(n)}\right)\right|_{\mathbb{U}_{X}^{n}} \simeq \operatorname{Ker}\left(\mathrm{K}_{X, \ell}^{(n)} \longrightarrow \mathrm{K}_{X, \ell-1}^{(n)}\right)\right|_{\mathbb{U}_{X}^{n}},
$$

where $\mathrm{K}_{X}^{(n)}=\mathrm{K}_{X, \bullet}^{(n)}=\alpha_{X}^{*}\left(\mathrm{~K}_{\mathbb{Z}}^{(n)}\right)$ (see Definition 6.3).

We consider now the cases $n$ odd and $n$ even separately.

8.1. The space $\mathbf{E}_{X}^{(n)}$ if $n=2 \ell+1$ is odd, that is, $r=0$ or $r=2$

Since the functor $(-)^{\vee}=\operatorname{Hom}_{A}(-, A)$ is left exact, we have

$$
S^{\vee}=\operatorname{Ker}\left(K_{\ell+1}^{\vee} \stackrel{d_{\ell+2}^{\vee}}{\longrightarrow} K_{\ell+2}^{\vee}\right)
$$

and hence a well-defined homomorphism

$$
\varphi_{\mathbb{Z}}^{(n)}:=\left.(-1)^{\ell} \epsilon_{n} \cdot \theta_{\ell} \cdot \bar{d}_{\ell+1}\right|_{\mathbb{U}_{\mathbb{Z}}^{n}}: \mathcal{E}_{\mathbb{Z}}^{(n)} \longrightarrow \mathcal{H o m}_{\mathcal{O}_{\mathbb{U}_{\mathbb{Z}}^{n}}}\left(\mathcal{E}_{\mathbb{Z}}^{(n)}, \mathcal{O}_{\mathbb{U}_{\mathbb{Z}}^{n}}\right)=\left.S^{\vee}\right|_{\mathbb{U}_{\mathbb{Z}}^{n}}
$$

which is $(-1)^{\ell}$-symmetric, where $\bar{d}_{\ell+1}$ is the unique morphism $S \longrightarrow K_{\ell}$, such that $d_{\ell+1}=\bar{d}_{\ell+1} \cdot \operatorname{pr}_{S}($ cf. (5)). We set

$$
\left(F_{\mathbb{Z}}^{(n)}, \phi_{\mathbb{Z}}^{(n)}\right):= \begin{cases}c_{0}\left(\mathcal{E}_{\mathbb{Z}}^{(n)}, \varphi_{\mathbb{Z}}^{(n)}\right) & \text { if } \ell \text { is even } \\ c_{0}\left(\mathcal{E}_{\mathbb{Z}}^{(n)}, \varphi_{\mathbb{Z}}^{(n)}\right)[1] & \text { if } \ell \text { is odd. }\end{cases}
$$


This is a symmetric $r$-pair. Recall now from 5.1.1-5.1.4 that the projection $\operatorname{pr}_{S}: K_{\ell+1} \rightarrow S$ induces a quasi-isomorphism (hence an isomorphism in $\mathbb{D}^{\mathrm{b}}\left(\mathrm{VB}_{A}\right)$ )

$$
\left.\left.p\right|_{\mathbb{U}_{\mathbb{Z}}^{n}}=\left.p(\mathbb{Z}, \underline{T})\right|_{\mathbb{U}_{\mathbb{Z}}^{n}}: \mathrm{C}_{\mathbb{Z}}^{(n)} \stackrel{\simeq}{\longrightarrow} c_{0}\left(\mathcal{E}_{\mathbb{Z}}^{(n)}\right) \quad \text { (respectively } \mathrm{C}_{\mathbb{Z}}^{(n)} \stackrel{\simeq}{\longrightarrow} c_{0}\left(\mathcal{E}_{\mathbb{Z}}^{(n)}\right)[1]\right),
$$

which is easily seen to be an isometry $\mathrm{E}_{\mathbb{Z}}^{(n)} \stackrel{\simeq}{\rightarrow}\left(F_{\mathbb{Z}}^{(n)}, \phi_{\mathbb{Z}}^{(n)}\right)$. It follows that $\phi_{\mathbb{Z}}^{(n)}$ is an isomorphism and so $\left(F_{\mathbb{Z}}^{(n)}, \phi_{\mathbb{Z}}^{(n)}\right)$ is a symmetric $r$-space. In particular, $\left(\mathcal{E}_{\mathbb{Z}}^{(n)}, \varphi_{\mathbb{Z}}^{(n)}\right)$ is a $(-1)^{\ell}$-symmetric space over $\mathbb{U}_{\mathbb{Z}}^{n}$. Applying the pull-back $v_{X}^{*}$ we get the following:

(i) the pair

$$
\left(\mathcal{E}_{X}^{(n)}, \varphi_{X}^{(n)}\right):=v_{X}^{*}\left(\mathcal{E}_{\mathbb{Z}}^{(n)}, \varphi_{\mathbb{Z}}^{(n)}\right)
$$

is a $(-1)^{\ell}$-symmetric space over $\mathbb{U}_{X}^{n}$;

(ii) the half Koszul space $\mathbf{E}_{X}^{(n)}$ is isometric to the short symmetric $r$-space:

$$
\mathbf{F}_{X}^{(n)}:=\left(F_{X}^{(n)}, \phi_{X}^{(n)}\right):=v_{X}^{*}\left(F_{\mathbb{Z}}^{(n)}, \phi_{\mathbb{Z}}^{(n)}\right)= \begin{cases}c_{0}\left(\mathcal{E}_{X}^{(n)}, \varphi_{X}^{(n)}\right) & \text { if } \ell \text { is even, } \\ c_{0}\left(\mathcal{E}_{X}^{(n)}, \varphi_{X}^{(n)}\right)[1] & \text { if } \ell \text { is odd. }\end{cases}
$$

8.2. The space $\mathbf{E}_{X}^{(n)}$ if $n=2 \ell$ is even, that is, $r=-1$ or $r=1$

We fix $L, M \subset K_{\ell}$ and $\lambda: L \stackrel{\simeq}{\rightarrow} M^{\vee}$ as in Lemma 5.1 (with $R=\mathbb{Z}$ ), and let $\operatorname{pr}_{L}: K_{\ell} \rightarrow L$ and $\operatorname{pr}_{M}: K_{\ell} \rightarrow M$ be the respective projections. We denote $\mathcal{L}:=\left.L\right|_{\mathbb{U}_{\mathbb{Z}}^{n}}$ and $\operatorname{pr}_{\mathcal{L}}:=\left.\operatorname{pr}_{L}\right|_{\mathbb{U}_{\mathbb{Z}}^{n}}:\left.K_{\ell}\right|_{\mathbb{U}_{\mathbb{Z}}^{n}} \rightarrow \mathcal{L}$.

On the complex $F_{\mathbb{Z}}^{(n)}:=\left.F_{\bullet}(A, \underline{T})\right|_{\mathbb{U}_{\mathbb{Z}}^{n}}$ we have the following symmetric $r$-form:

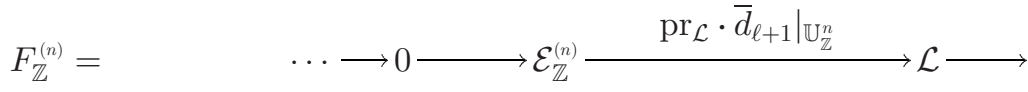

$$
\begin{aligned}
& \phi_{\mathbb{Z}}^{(n)} \downarrow:=\left.\quad \downarrow \epsilon_{n} \bar{h}\right|_{\mathbb{U}_{\mathbb{Z}}^{n}} \downarrow \quad(-1)^{\ell} \epsilon_{n}\left(\left.\bar{h}\right|_{\mathbb{U}_{\mathbb{Z}}^{n}}\right)^{\vee} \operatorname{can}_{\mathcal{L}} \downarrow \\
& \mathcal{D}_{\mathbb{U}_{\mathbb{Z}}^{n}}\left(F_{\mathbb{Z}}^{(n)}\right)\left[(-1)^{\ell+1}\right]=\quad \cdots \longrightarrow 0 \longrightarrow \mathcal{L}^{\vee} \underset{\left(\left.\operatorname{pr}_{\mathcal{L}} \cdot \bar{d}_{\ell+1}\right|_{\mathbb{U}_{\mathbb{Z}}^{n}}\right)^{\vee}}{\longrightarrow}\left(\mathcal{E}_{\mathbb{Z}}^{(n)}\right)^{\vee} \longrightarrow \\
& \text { if } r=-1 \quad \operatorname{deg} 0 \quad \operatorname{deg}-1 \\
& \text { if } r=1 \quad \operatorname{deg} 1 \quad \operatorname{deg} 0
\end{aligned}
$$

where $\bar{h}=\lambda \cdot \operatorname{can}_{M} \cdot \operatorname{pr}_{M} \cdot \bar{d}_{\ell+1}$. Since $\left.d_{\ell+1}\right|_{\mathbb{U}_{\mathbb{Z}}^{n}}=\left.\left(\bar{d}_{\ell+1} \cdot \operatorname{pr}_{S}\right)\right|_{\mathbb{U}_{\mathbb{Z}}^{n}}$, we see that the quasi-isomorphism $\left.p\right|_{\mathbb{U}_{\mathbb{Z}}^{n}}$ : $\mathrm{C}_{\mathbb{Z}}^{(n)} \stackrel{\simeq}{\rightarrow} F_{\mathbb{Z}}^{(n)}$ is an isometry $\mathbf{E}_{\mathbb{Z}}^{(n)} \stackrel{\simeq}{\rightarrow}\left(F_{\mathbb{Z}}^{(n)}, \phi_{\mathbb{Z}}^{(n)}\right)$, and so $\phi_{\mathbb{Z}}^{(n)}$ is an isomorphism in $\mathbb{D}^{\mathrm{b}}\left(\mathrm{VB}_{\mathbb{Z}}\right)$. Therefore $\left(F_{\mathbb{Z}}^{(n)}, \phi_{\mathbb{Z}}^{(n)}\right)$ is a symmetric $r$-space over $\mathbb{U}_{\mathbb{Z}}^{n}$. Applying the pull-back $v_{X}^{*}$ we see that the half Koszul space $\mathbf{E}_{X}^{(n)}$ is isometric to the short symmetric $r$-space

$$
\mathbf{F}_{X}^{(n)}:=\left(F_{X}^{(n)}, \phi_{X}^{(n)}\right):=v_{X}^{*}\left(F_{\mathbb{Z}}^{(n)}, \phi_{\mathbb{Z}}^{(n)}\right) .
$$

\section{Witt groups of the punctured affine space}

Recall the notation of $\S 2$, like formula (3), defining $r \in\{-1,0,1,2\}$ by $n=4 q+r+1$. We begin with an easy application of triangular Witt theory.

Theorem 9.1. Let $X$ be a regular scheme containing $\frac{1}{2}$. There exists a split short exact sequence

$$
0 \longrightarrow \mathrm{W}^{i}(X) \stackrel{\sigma_{X}^{*}}{\longrightarrow} \mathrm{W}^{i}\left(\mathbb{U}_{X}^{n}\right) \stackrel{\partial}{\longrightarrow} \mathrm{W}_{X}^{i+1}\left(\mathbb{A}_{X}^{n}\right) \longrightarrow 0,
$$


for all $i \in \mathbb{Z}$, where $\partial$ is the connecting homomorphism of the localization $\mathbb{U}_{X}^{n} \subset \mathbb{A}_{X}^{n}$. This sequence is natural in $X$ in the obvious way. Moreover, a left inverse to $\sigma_{X}^{*}$ is given by $\gamma^{*}: \mathrm{W}^{i}\left(\mathbb{U}_{X}^{n}\right) \rightarrow \mathrm{W}^{i}(X)$ for any $X$-point $\gamma: X \rightarrow \mathbb{U}_{X}^{n}$, that is, any morphism $\gamma: X \rightarrow \mathbb{U}_{X}^{n}$ such that $\sigma_{X} \circ \gamma=\mathrm{id}_{X}$.

Proof. This result follows from the localization sequence [2] and homotopy invariance $[4]$.

We want to apply 'dévissage' to the relative groups $\mathrm{W}_{X}^{i+1}\left(\mathbb{A}_{X}^{n}\right)$ and we will need the following theorem.

TheOREM 9.2. Let $X$ be a regular $\mathbb{Z}[1 / 2]$-scheme of finite Krull dimension. Consider the structure morphism $\pi_{X}: \mathbb{A}_{X}^{n} \rightarrow X$. Then, the homomorphism

$$
\vartheta_{X}^{(n)}: \mathrm{W}^{i-n}(X) \longrightarrow \mathrm{W}_{X}^{i}\left(\mathbb{A}_{X}^{n}\right), \quad w \longmapsto \pi_{X}^{*}(w) \star \kappa_{X}^{(n)}
$$

is an isomorphism for all $i \in \mathbb{Z}$.

Proof. The affine case $X=\operatorname{Spec} R$ is [10, Theorem 9.3] and the global case follows from this using the Mayer-Vietoris exact sequence [4, Theorem 2.6].

REMARK 9.3. We do not know whether $\vartheta_{X}^{(n)}$ is an isomorphism for more general schemes like, for example, regular schemes of infinite Krull dimension. The proof of [10, Theorem 9.3] uses coherent Witt theory and therefore only applies to regular rings of finite Krull dimension.

Theorem 9.4. Let $X$ be a $\mathbb{Z}[1 / 2]$-scheme. Let $1 \leqslant i \leqslant n$ be an integer and consider the $X$-point $\gamma_{i}: X \rightarrow \mathbb{U}_{X}^{n} \subset \mathbb{A}_{X}^{n}$ corresponding to $T_{i}=1$ and $T_{j}=0$ for all $j \neq i$. If $n \geqslant 2$, then the evaluation at this point of the Witt class $\varepsilon_{X}^{(n)} \in \mathrm{W}^{r}\left(\mathbb{U}_{X}^{n}\right)$ of the half-Koszul space is zero,

$$
\gamma_{i}^{*}\left(\varepsilon_{X}^{(n)}\right)=0 \quad \text { in } \mathrm{W}^{r}(X)
$$

Proof. Consider the 'same' point $\gamma_{i}=(0, \ldots, 0,1,0, \ldots, 0)$ but over $\mathbb{Z}[1 / 2]$ instead of $X$, that is, $\gamma_{i}: \operatorname{Spec}(\mathbb{Z}[1 / 2]) \rightarrow \mathbb{U}_{\mathbb{Z}[1 / 2]}^{n}$. We have a commutative diagram

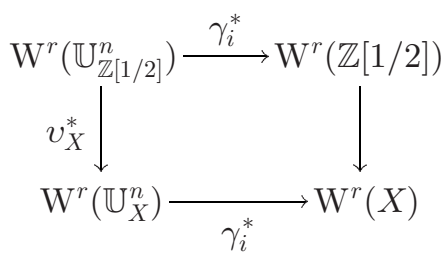

with the obvious morphisms, and we know from Lemma 7.5 that $v_{X}^{*}\left(\varepsilon_{\mathbb{Z}[1 / 2]}^{(n)}\right)=\varepsilon_{X}^{(n)}$. Therefore, it suffices to prove the result for $\mathbb{Z}[1 / 2]$.

If $r \neq 0$ this is trivially true because in this case $\mathrm{W}^{r}(\mathbb{Z}[1 / 2])=0$. In fact, since $\mathbb{Z}[1 / 2]$ is a Dedekind domain, we have, by $[6$, Theorem 10.1],

$$
\mathrm{W}^{-1}(\mathbb{Z}[1 / 2])=\mathrm{W}^{2}(\mathbb{Z}[1 / 2])=0
$$


and

$$
\mathrm{W}^{1}(\mathbb{Z}[1 / 2]) \simeq \operatorname{Coker}\left(\mathrm{W}(\mathbb{Z}[1 / 2]) \stackrel{\sum \partial_{p}}{\longrightarrow} \bigoplus_{p \neq 2} \mathrm{~W}(\mathbb{Z} / \mathbb{Z} p)\right),
$$

where $\partial_{p}$ is a second residue homomorphism associated with the prime number $p$. But this cokernel is also zero by the classical calculation of the Witt group of $\mathbb{Q}$, cf. for example, [17, Theorem VI.6.11].

If $r=0$, that is, $n=2 \ell+1$ with $\ell \neq 0$ even, this follows from the next lemma.

LEMma 9.5. Let $R$ be a ring and $n=2 \ell+1$ with $\ell \geqslant 2$ even. Then $\gamma_{i}^{*}\left(\mathbf{E}_{R}^{(n)}\right) \simeq$ $\gamma_{i}^{*}\left(\mathcal{E}_{R}^{(n)}, \varphi_{R}^{(n)}\right)$ is trivial in $\mathrm{W}(R) \simeq \mathrm{W}^{0}(R)$.

Proof. After renumbering we may assume $i=1$. Let $K_{\mathbf{0}}=K_{\mathbf{0}}(A, \underline{T})$ be the Koszul complex of the regular sequence $\underline{T}=\left(T_{1}, \ldots, T_{n}\right)$ over $A=R\left[T_{1}, \ldots, T_{n}\right]$, and $\Theta=\Theta(A, \underline{T}): K_{.} \stackrel{\simeq}{\rightarrow} \mathcal{D}_{A}\left(K_{\text {. }}\right)[n]$ the canonical symmetric $n$-form. Recall that the differential $d_{s}: K_{s} \rightarrow K_{s-1}$ is then given by

$$
e_{i_{1}} \wedge \ldots \wedge e_{i_{s}} \longmapsto \sum_{j=1}^{s}(-1)^{j-1} T_{i_{j}} \cdot e_{i_{1}} \wedge \ldots \wedge e_{i_{j-1}} \wedge e_{i_{j+1}} \wedge \ldots \wedge e_{i_{s}}
$$

where $e_{1}, \ldots, e_{n}$ constitute a basis of $A^{n}$. We denote by $\iota_{R}$ the open immersion $\mathbb{U}_{R}^{n} \hookrightarrow \mathbb{A}_{R}^{n}$ (cf. (3)). Then we have $\gamma_{1}^{*}\left(\mathbf{E}_{R}^{(n)}\right)=\gamma_{1}^{*} \iota_{R}^{*}\left(\mathbf{C}_{R}^{(n)}\right)$ and $K_{\bullet}^{\prime}=\gamma_{1}^{*} \iota_{R}^{*}\left(K_{\bullet}\right)$ is the Koszul complex $K_{.}(R, \underline{t})$ for the sequence $\underline{t}=(1,0, \ldots, 0) \subset R$. We denote the differential of this Koszul complex by $d^{\prime}$. Note that this complex is split exact. The isomorphism of complexes $\Theta^{\prime}:=\gamma_{1}^{*} \iota_{R}^{*}(\Theta)$ is a symmetric $n$-form on $K_{\text {! }}^{\prime}$ and

$$
\left(C^{\prime}, \Xi^{\prime}\right):=\gamma_{1}^{*} \iota_{R}^{*}\left(\mathbf{C}_{R}^{(n)}\right)
$$

is the symmetric 0-pair $(C .(R, \underline{t}), \Xi(R, \underline{t}))$ (see 5.1.1) which is in fact a symmetric 0 -space since $K_{\bullet}^{\prime}[-2 q]=$ cone $\Xi(R, \underline{t})$ is split exact.

We give now a direct summand $S^{\prime}$ of $K_{\ell+1}^{\prime}$, such that the projection $K_{\ell+1}^{\prime} \rightarrow S^{\prime}$ induces a quasi-isomorphism $C_{\bullet}^{\prime} \stackrel{\simeq}{\rightarrow} c_{0}\left(S^{\prime}\right)$, where $c_{0}: \mathrm{VB}_{R} \rightarrow \mathbb{D}^{\mathrm{b}}\left(\mathrm{VB}_{R}\right)$ is the natural embedding.

The elements $v_{i}=1 \otimes e_{i}(i=1, \ldots, n)$ are a basis of $R \otimes_{A} A^{n}=\gamma_{1}^{*} \iota_{R}^{*}\left(A^{n}\right)$, and so the exterior products $v_{i_{1}} \wedge \ldots \wedge v_{i_{s}}\left(1 \leqslant i_{1}<\ldots<i_{s} \leqslant n\right)$ are free generators of $K_{s}^{\prime} \simeq \bigwedge^{s} R^{n}$. The differential $d_{s}^{\prime}$ acts on them as follows:

$$
d_{s}^{\prime}\left(v_{i_{1}} \wedge \ldots \wedge v_{i_{s}}\right)= \begin{cases}0 & \text { for } 2 \leqslant i_{1}<\ldots<i_{s} \leqslant n \\ v_{i_{2}} \wedge \ldots \wedge v_{i_{s}} & \text { for } 1=i_{1}<i_{2}<\ldots<i_{s} \leqslant n .\end{cases}
$$

Therefore the $R$-module $S^{\prime}:=v_{1} \wedge\left(\bigwedge^{\ell} R^{n}\right) \subset K_{\ell+1}^{\prime}$ is isomorphic to Coker $d_{\ell+2}^{\prime}$.

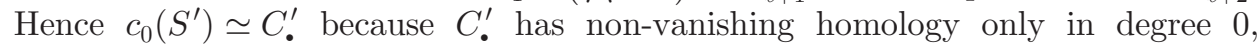
$C_{0}^{\prime}=K_{\ell+1}^{\prime}$ and $C_{i}^{\prime}=0$ for $i<0$. We get an isometry $c_{0}\left(S^{\prime}, \varphi^{\prime}\right) \simeq\left(C^{\prime}, \Xi^{\prime}\right)$, where $\varphi^{\prime}:=\left.\epsilon_{n}\left(\Theta_{l}^{\prime} \cdot d_{\ell+1}^{\prime}\right)\right|_{S^{\prime}}$.

Consider now the following free submodule $M^{\prime}:=\left(v_{1} \wedge v_{2}\right) \wedge\left(\wedge^{\ell-1} R^{n}\right)$ of $S^{\prime}$. We claim that $M^{\prime}$ is a totally isotropic subspace of $\left(S^{\prime}, \varphi^{\prime}\right)$. From this the lemma follows because $\operatorname{rank} M^{\prime}=\frac{1}{2} \operatorname{rank} S^{\prime}$ and so $\left(S^{\prime}, \varphi^{\prime}\right)$ is hyperbolic by [1, I, Theorem 4.6].

To see this we use the description of $\Theta$ given in Remark 4.2. Let $\omega: \bigwedge^{n} A^{n} \cong A$ be as in this remark and $\omega^{\prime}:=\operatorname{id}_{R} \otimes \omega$. Then $\omega^{\prime}\left(v_{1} \wedge \ldots \wedge v_{n}\right)=1$ and

$$
\Theta_{\ell}^{\prime}(x)(y)= \pm \omega^{\prime}(x \wedge y) \quad \text { for all } x \in K_{\ell}^{\prime} \text { and } y \in K_{\ell+1}^{\prime} \text {. }
$$


If now $x, y \in M^{\prime}$ then $y=v_{1} \wedge v_{2} \wedge y^{\prime}$ and $d_{\ell+1}^{\prime}(x)=v_{2} \wedge x^{\prime}$ for some $x^{\prime}, y^{\prime} \in \wedge^{\ell-1} R^{n}$, and so $\pm \varphi^{\prime}(x)(y)=\omega^{\prime}\left(d_{\ell+1}^{\prime}(x) \wedge y\right)=0$ since $v_{2} \wedge v_{2}=0$.

TheOREM 9.6. Let $X$ be a regular $\mathbb{Z}[1 / 2]$-scheme. The composition of the connecting homomorphism with the 4-periodicity isomorphism,

$$
\mathrm{W}^{r}\left(\mathbb{U}_{X}^{n}\right) \stackrel{\partial}{\longrightarrow} \mathrm{W}_{X}^{r+1}\left(\mathbb{A}_{X}^{n}\right) \stackrel{\tau^{q}}{\longrightarrow} \mathrm{W}_{X}^{n}\left(\mathbb{A}_{X}^{n}\right),
$$

maps the Witt class $\varepsilon_{X}^{(n)} \in \mathrm{W}^{r}\left(\mathbb{U}_{X}^{n}\right)$ of the half-Koszul space to the Witt class $\kappa_{X}^{(n)} \in \mathrm{W}_{X}^{n}\left(\mathbb{A}_{X}^{n}\right)$ of the Koszul space over $\mathbb{A}_{X}^{n}$.

Proof. Recall that we always have $n=4 q+r+1$. The statement is a direct consequence of Theorem 7.3, using the definition of the connecting homomorphism $\partial$ via the symmetric cone and the fact that $\varepsilon_{X}^{(n)}=\left[\left.\mathbf{C}_{X}^{(n)}\right|_{\mathbb{U}_{X}^{n}}\right]$ by Definition 7.4.

TheOREM 9.7. Let $X$ be a regular $\mathbb{Z}[1 / 2]$-scheme. For all $i \in \mathbb{Z}$, define the following homomorphism:

$$
\rho_{X}^{(n)}: \mathrm{W}^{i-r}(X) \longrightarrow \mathrm{W}^{i}\left(\mathbb{U}_{X}^{n}\right), \quad w \longmapsto \sigma_{X}^{*}(w) \star \varepsilon_{X}^{(n)} .
$$

Then the diagram

$$
\begin{gathered}
\mathrm{W}^{i-r}(X) \stackrel{\tau^{q}}{\simeq} \mathrm{W}^{i+1-n}(X) \\
\left.\rho_{X}^{(n)}\right|^{\simeq} \underset{\vartheta_{X}^{(n)}}{ } \\
\mathrm{W}^{i}\left(\mathbb{U}_{X}^{n}\right) \stackrel{\partial}{\longrightarrow} \mathrm{W}_{X}^{i+1}\left(\mathbb{A}_{X}^{n}\right)
\end{gathered}
$$

commutes, for all $i \in \mathbb{Z}$, where the isomorphism $\vartheta_{X}^{(n)}$ is the one of Theorem 9.2 and where $\tau$ is the 4-periodicity isomorphism.

Proof. Recall of course that $r=n-4 q-1$ by (1). We have to show that

$$
\partial \rho_{X}^{(n)}([x])=\vartheta_{X}^{(n)}\left(\tau^{-q}([x])\right)
$$

for all $[x] \in \mathrm{W}^{i-r}(X)$. Using the fact that $\sigma_{X}: \mathbb{U}_{X}^{n} \rightarrow X$ factors as

$$
\mathbb{U}_{X}^{n} \stackrel{\iota_{X}}{\longrightarrow} \mathbb{A}_{X}^{n} \stackrel{\pi_{X}}{\longrightarrow} X
$$

we get

$$
\begin{aligned}
\partial \rho_{X}^{(n)}([x]) & =\partial\left(\iota_{X}^{*}\left(\pi_{X}^{*}([x])\right) \star \varepsilon_{X}^{(n)}\right) & & \\
& =\pi_{X}^{*}([x]) \star \partial\left(\varepsilon_{X}^{(n)}\right) & & \text { (by }[\mathbf{1 1}, \text { Theorem 2.11]) } \\
& =\pi_{X}^{*}([x]) \star \tau^{-q}\left(\kappa_{X}^{(n)}\right) & & \text { (by Theorem 7.3). }
\end{aligned}
$$

But this is equal to the right-hand side of (11) because

$$
\begin{aligned}
\vartheta_{X}^{(n)}\left(\tau^{-q}([x])\right) & =\tau^{-q}\left(\pi_{X}^{*}([x])\right) \star \kappa_{X}^{(n)} & & \text { (by Lemma B.1) } \\
& =\pi_{X}^{*}([x]) \star \tau^{-q}\left(\kappa_{X}^{(n)}\right) & & \text { (by Lemma B.3) }
\end{aligned}
$$

Corollary 9.8 . Let $X$ be a regular $\mathbb{Z}[1 / 2]$-scheme. We have an isomorphism for all $i \in \mathbb{Z}$.

$$
\left(\sigma_{X}^{*}, \rho_{X}^{(n)}\right): \mathrm{W}^{i}(X) \oplus \mathrm{W}^{i-r}(X) \stackrel{\simeq}{\longrightarrow} \mathrm{W}^{i}\left(\mathbb{U}_{X}^{n}\right)
$$


Proof. Comparing the split exact sequence of Theorem 9.1 and the obvious split exact sequence $0 \rightarrow \mathrm{W}^{i}(X) \rightarrow \mathrm{W}^{i}(X) \oplus \mathrm{W}^{i-r}(X) \rightarrow \mathrm{W}^{i-r}(X) \rightarrow 0$, we need only note that $\partial \cdot \rho_{X}^{(n)}$ is an isomorphism, as follows from Theorem 9.7.

To understand the ring structure on $\mathrm{W}^{\text {tot }}\left(\mathbb{U}_{X}^{n}\right)$, we need some properties of the symmetric spaces $\mathbf{K}_{X}^{(n)}$ and $\mathbf{E}_{X}^{(n)}$, which can also be proven for schemes that are not necessarily regular. The case $n=1$, that is, the 'Laurent scheme' case, is well known, so we have to deal with $n \geqslant 2$.

Theorem 9.9. Let $X$ be a $\mathbb{Z}[1 / 2]$-scheme. If $n \geqslant 2$ then the symmetric $r$-space $\mathbf{E}_{X}^{(n)}$ is locally trivial, that is, for any $x \in \mathbb{U}_{X}^{n}$ we have $\left[\left(\mathbf{E}_{X}^{(n)}\right)_{x}\right]=0$ in $\mathrm{W}^{r}\left(\mathcal{O}_{\mathbb{U}_{X}^{n}, x}\right)$.

Proof. Define for all $i \in\{1, \ldots, n\}$ the principal open $\mathbb{V}_{X}^{n}(i)$ of $\mathbb{A}_{X}^{n}$ given by the equation $T_{i} \neq 0$. Let $J \subseteq\{1, \ldots, n\} \subset \mathbb{N}$. We define

$$
\mathbb{V}_{X}^{n}(J):=\bigcup_{j \in J} \mathbb{V}_{X}^{n}(j) \subseteq \mathbb{U}_{X}^{n}=\mathbb{V}_{X}^{n}(\{1, \ldots, n\})
$$

and denote the corresponding open immersion by $\iota_{J}: \mathbb{V}_{X}^{n}(J) \rightarrow \mathbb{U}_{X}^{n}$. Since $n \geqslant 2$, we can cover $\mathbb{U}_{X}^{n}$ with the open subschemes $\mathbb{V}_{X}^{n}(J)$ with $|J| \leqslant n-1$. So it suffices to prove the following stronger result.

TheOREM 9.10. With the above notation, if $|J| \leqslant(n-1)$ then $\left[\left.\mathbf{E}_{X}^{(n)}\right|_{\mathbb{V}_{X}^{n}(J)}\right]=0$ in $\mathrm{W}^{r}\left(\mathbb{V}_{X}^{n}(J)\right)$.

Proof. We easily reduce to the case $X=\operatorname{Spec} \mathbb{Z}[1 / 2]$. In this case we argue as follows. For brevity we set $R:=\mathbb{Z}[1 / 2]$.

For $J$ empty, the result is trivial since $\mathbb{V}_{R}^{n}(J)=\emptyset$ and its Witt group is zero. So we assume that $J \neq \emptyset$. Consider the closed complement $\mathbb{Y}_{R}(J):=\mathbb{A}_{R}^{n} \backslash \mathbb{V}_{R}^{n}(J)$ of $\mathbb{V}_{R}^{n}(J) \subset \mathbb{U}_{R}^{n}$. Note that $\mathbb{A}_{X}^{n} \backslash \mathbb{U}_{R}^{n} \subset \mathbb{Y}_{R}(J)$. By Theorem 9.1, we have the following commutative diagram with exact rows:

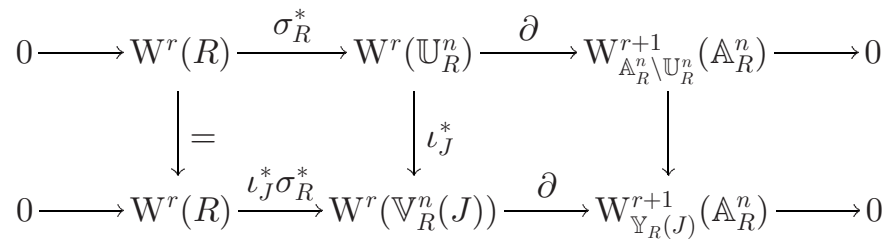

We get from the right-hand commutative square, from Theorem 9.6, and from Proposition 4.5 that $\partial\left(\iota_{J}^{*}\left(\varepsilon_{R}^{(n)}\right)\right)=0$. Therefore, by exactness of the above second row, there exists a unique class $w \in \mathrm{W}^{r}(R)$ such that $\iota_{J}^{*}\left(\varepsilon_{R}^{(n)}\right)=\iota_{J}^{*}\left(\sigma_{R}^{*}(w)\right)$. In fact, $w=\gamma^{*}\left(\iota_{J}^{*}\left(\varepsilon_{R}^{(n)}\right)\right)$ for any $R$-point $\gamma: \operatorname{Spec}(R) \rightarrow \mathbb{V}_{R}^{n}(J)$, which exists by the assumption $J \neq \emptyset$. Choose $j \in J$ and define the $R$-point $\gamma: \operatorname{Spec}(R) \rightarrow \mathbb{V}_{R}^{n}(J)$ to be given by $T_{j}=1$ and $T_{i}=0$ for $i \neq j$. Since $w=\gamma^{*}\left(\iota_{J}^{*}\left(\varepsilon_{R}^{(n)}\right)\right)=\left(\iota_{J} \cdot \gamma\right)^{*}\left(\varepsilon_{R}^{(n)}\right)$ and since $\iota_{J} \cdot \gamma: \operatorname{Spec}(R) \rightarrow \mathbb{U}_{R}^{n}$ is simply the $R$-point $\gamma_{j}$ of Theorem 9.4 , we conclude from it that $w=0$. Hence $\iota_{J}^{*}\left(\varepsilon_{R}^{(n)}\right)=0$ as wanted.

REMARK 9.11. The statement of Theorem 9.9 is obviously not true for $n=1$. The proof fails for $n=1$ because then $\mathbb{V}_{X}^{n}(J)=\emptyset$ for any $J$ such that $|J| \leqslant n-1=0$ and hence we cannot cover $\mathbb{U}_{X}^{n}$ with these. 
It follows from this theorem above and [5, Theorem 4.2] that if $n \geqslant 2$ the space $\varepsilon_{X}^{(n)}$ is nilpotent in $\mathrm{W}^{\text {tot }}\left(\mathbb{U}_{X}^{n}\right)$. We prove a more precise result.

Theorem 9.12. Let $X$ be a $\mathbb{Z}[1 / 2]$-scheme. Assume that $n \geqslant 2$. Then

$$
\left(\varepsilon_{X}^{(n)}\right)^{2}=\varepsilon_{X}^{(n)} \star \varepsilon_{X}^{(n)}=0
$$

in $\mathrm{W}^{\text {tot }}\left(\mathbb{U}_{X}^{n}\right)$. If $n=1$ then $\left(\varepsilon_{X}^{(n)}\right)^{2}=1$ in $\mathrm{W}^{\text {tot }}\left(\mathbb{U}_{X}^{n}\right)$.

Proof. The case $n=1$ is classical, so let $n \geqslant 2$. Since

$$
v_{X}^{*}: \mathrm{W}^{\text {tot }}\left(\mathbb{U}_{\mathbb{Z}[1 / 2]}^{n}\right) \rightarrow \mathrm{W}^{\text {tot }}\left(\mathbb{U}_{X}^{n}\right)
$$

is a morphism of graded rings (cf. [11, Theorem 3.2]) and $v_{X}^{*}\left(\varepsilon_{\mathbb{Z}[1 / 2]}^{(n)}\right)=\varepsilon_{X}^{(n)}(\mathrm{cf}$. Lemma 7.5) it is enough to prove this for the affine scheme $X=\operatorname{Spec} \mathbb{Z}[1 / 2]$.

Because we assume $n \geqslant 2$ there exist non-empty subsets $J_{1}, J_{2} \subset\{1, \ldots, n\}$ with $J_{1} \neq J_{2}$ and $J_{1} \cup J_{2}=\{1, \ldots, n\}$. We define $\mathbb{V}_{\mathbb{Z}[1 / 2]}^{n}\left(J_{i}\right) \subseteq \mathbb{U}_{\mathbb{Z}[1 / 2]}^{n}$ as in the proof of Theorem 9.9 above and let $\mathbb{Y}_{\mathbb{Z}[1 / 2]}^{n}\left(J_{i}\right):=\mathbb{U}_{\mathbb{Z}[1 / 2]}^{n} \backslash \mathbb{V}_{\mathbb{Z}[1 / 2]}^{n}\left(J_{i}\right)$ be the complement $(i=1,2)$. Note that $J_{1} \cup J_{2}=\{1, \ldots, n\}$ implies $\mathbb{Y}_{\mathbb{Z}[1 / 2]}^{n}\left(J_{1}\right) \cap \mathbb{Y}_{\mathbb{Z}[1 / 2]}^{n}\left(J_{2}\right)=\emptyset$.

By Theorem 9.10 we know that $\left[\left.\mathbf{E}_{\mathbb{Z}[1 / 2]}^{(n)}\right|_{\mathbb{Z}[1 / 2]} ^{n}\left(J_{i}\right)\right]=0$ for $i=1,2$. Therefore by the localization sequence there exists $x_{i} \in \mathrm{W}_{\mathbb{Y}_{\mathbb{Z}[1 / 2]}^{n}\left(J_{i}\right)}^{r}\left(\mathbb{U}_{\mathbb{Z}[1 / 2]}^{n}\right)$ with $x_{i}=\varepsilon_{\mathbb{Z}[1 / 2]}^{(n)}$ in $\mathrm{W}^{r}\left(\mathbb{U}_{\mathbb{Z}[1 / 2]}^{n}\right)$ for $i=1,2$, and so $x_{1} \star x_{2}=\left(\varepsilon_{\mathbb{Z}[1 / 2]}^{(n)}\right)^{2}$ in $\mathrm{W}^{2 r}\left(\mathbb{U}_{\mathbb{Z}[1 / 2]}^{n}\right)$. But the space $x_{1} \star x_{2}$ lives on a complex with support in $\mathbb{Y}_{\mathbb{Z}[1 / 2]}^{n}\left(J_{1}\right) \cap \mathbb{Y}_{\mathbb{Z}[1 / 2]}^{n}\left(J_{2}\right)=\emptyset$ and so $\left(\varepsilon_{\mathbb{Z}[1 / 2]}^{(n)}\right)^{2}=0$.

Denote by $\mathrm{W}^{\text {tot }}(X)[\varepsilon]$ the graded skew polynomial ring in one variable $\varepsilon$ of degree $r$ over the graded ring $\mathrm{W}^{\text {tot }}(X)$. Recall that this means that $c \cdot \varepsilon=(-1)^{r \operatorname{deg} c}(\varepsilon \cdot c)$ for a homogeneous element $c \in \mathrm{W}^{\text {tot }}(X)$. We have a homogeneous homomorphism of graded rings given by

$$
\mathrm{W}^{\mathrm{tot}}(X)[\varepsilon] \longrightarrow \mathrm{W}^{\mathrm{tot}}\left(\mathbb{U}_{X}^{n}\right), \quad \sum_{i=0}^{m} c_{i} \varepsilon^{i} \longmapsto \sum_{i=0}^{m} \sigma_{X}^{*}\left(c_{i}\right) \star\left(\varepsilon_{X}^{(n)}\right)^{i} .
$$

Using this morphism we can restate Corollary 9.8 and Theorem 9.12 as follows.

TheOREM 9.13. Let $X$ be a regular scheme of finite Krull dimension over $\mathbb{Z}[1 / 2]$. Then we have an isomorphism of graded rings:

$$
\left.\begin{array}{l}
\text { if } n \geqslant 2, \quad \mathrm{~W}^{\mathrm{tot}}(X)[\varepsilon] /\left(\varepsilon^{2}\right) \\
\text { if } n=1, \quad \mathrm{~W}^{\mathrm{tot}}(X)[\varepsilon] /\left(\varepsilon^{2}-1\right)
\end{array}\right\} \stackrel{\simeq}{\longrightarrow} \mathrm{W}^{\mathrm{tot}}\left(\mathbb{U}_{X}^{n}\right) .
$$

EXAMPLE 9.14. Let $R$ be a regular ring of finite Krull dimension which contains $\frac{1}{2}$ and

$$
\Sigma_{R}^{2 n-1}:=\operatorname{Spec}\left(R\left[T_{1}, \ldots, T_{n}, Y_{1}, \ldots, Y_{n}\right]\right) /\left(1-\sum_{i=1}^{n} T_{i} Y_{i}\right)
$$

be the hyperbolic $(2 n-1)$-sphere over $R$. The $R$-algebra morphism

$$
R\left[T_{1}, \ldots, T_{n}\right] \longrightarrow R\left[T_{1}, \ldots, T_{n}, Y_{1}, \ldots, Y_{n}\right] /\left(1-\sum_{i=1}^{n} T_{i} Y_{i}\right),
$$

which maps $T_{i}$ to $T_{i} \bmod 1-\sum T_{i} Y_{i}$, induces a flat morphism $f: \Sigma_{R}^{2 n-1} \rightarrow \mathbb{U}_{R}^{n}$ whose fibers are affine spaces over the appropriate residue fields. Therefore by strong homotopy invariance [9, Corollary 4.2] we get an isomorphism of graded 
Witt rings $f^{*}: \mathrm{W}^{\text {tot }}\left(\mathbb{U}_{R}^{n}\right) \stackrel{\simeq}{\rightarrow} \mathrm{W}^{\text {tot }}\left(\Sigma_{R}^{2 n-1}\right)$. In particular, $\mathrm{W}^{\text {tot }}\left(\Sigma_{R}^{2 n-1}\right)$ is also a free $\mathrm{W}^{\text {tot }}(R)$-module with two generators. This has been proven by Karoubi [13] if $R$ is the field of complex numbers.

Note that if $R$ is a regular domain and $R\left(\Sigma_{R}^{2 n-1}\right)$ is the function field of the hyperbolic sphere then Theorem 9.9 implies that the natural morphism

$$
\mathrm{W}\left(\Sigma_{R}^{2 n-1}\right) \longrightarrow \mathrm{W}\left(R\left(\Sigma_{R}^{2 n-1}\right)\right)
$$

is not injective.

\section{Witt non-triviality of the (half) Koszul spaces}

Theorem 10.1. Let $X$ be a scheme which is not of equicharacteristic 2. Then the Witt class of the symmetric n-space $\mathbf{K}_{X}^{(n)}$ is non-trivial in the Witt group with support $\mathrm{W}_{X}^{n}\left(\mathbb{A}_{X}^{n}\right)$.

Proof. By assumption, there is a point $x \in X$ whose residue field $k(x)$ has characteristic different from 2. By specialization at $x$ (see Remark 6.4 for naturality), it suffices to prove the result for the regular $\mathbb{Z}[1 / 2]$-scheme $X:=\operatorname{Spec}(k(x))$. Here, we apply Theorem 9.2 with $i:=n$ and $w:=1 \in \mathrm{W}^{0}(X)$, the unit of the Witt ring.

TheOREM 10.2. Let $X$ be any scheme which is not of equicharacteristic 2 . Then the Witt class $\varepsilon_{X}^{(n)}$ of the symmetric $r$-space $\mathbf{E}_{X}^{(n)}$ is not in the image of the natural homomorphism $\mathrm{W}^{r}\left(\mathbb{A}_{X}^{n}\right) \rightarrow \mathrm{W}^{r}\left(\mathbb{U}_{X}^{n}\right)$. In particular, $\mathbf{E}_{X}^{(n)}$ cannot be extended to the whole affine space $\mathbb{A}_{X}^{n}$.

Proof. Again, by specialization at a point $x$ with $\operatorname{char}(k(x)) \neq 2$, we are reduced to proving the result for the $\mathbb{Z}[1 / 2]$-regular scheme $X:=\operatorname{Spec}(k(x))$. In this case, the following composition vanishes:

$$
\mathrm{W}^{r}\left(\mathbb{A}_{X}^{n}\right) \stackrel{\iota_{X}^{*}}{\longrightarrow} \mathrm{W}^{r}\left(\mathbb{U}_{X}^{n}\right) \stackrel{\partial}{\longrightarrow} \mathrm{W}_{X}^{r+1}\left(\mathbb{A}_{X}^{n}\right) .
$$

Here, the connecting homomorphism $\partial$ is, for instance, as in Theorem 9.6, where we proved that $\partial\left(\varepsilon_{X}^{(n)}\right)$ coincides with $\left[\mathbf{K}_{X}^{(n)}\right]$, up to 4-periodicity. So, $\varepsilon_{X}^{(n)}$ cannot be extended to $\mathbb{A}_{X}^{n}$ since $\left[\mathbf{K}_{X}^{(n)}\right] \neq 0 \in \mathrm{W}_{X}^{n}\left(\mathbb{A}_{X}^{n}\right)$ by Theorem 10.1. Note that we pass via the regular case to use the connecting homomorphism $\partial$.

\section{Appendix A. The locally free module $\mathcal{E}_{X}^{(n)}$}

We use the notation of the main part of the text. We want to prove the following.

Theorem A.1. Let $X$ be a noetherian scheme and $n \geqslant 4$. Then there does not exist a locally free $\mathcal{O}_{\mathbb{A}_{X}^{n}}$-module $\mathcal{F}$ such that $\left.\mathcal{F}\right|_{\mathbb{U}_{X}^{n}} \simeq \mathcal{E}_{X}^{(n)}$. In particular, $\mathcal{E}_{X}^{(n)}$ is not a free $\mathcal{O}_{\mathbb{U}_{X}^{n}}$-module.

Let $x \in X$ and $\operatorname{Spec} k(x) \stackrel{f}{\rightarrow} X$ be the corresponding point. If there exists a locally free $\mathcal{O}_{\mathbb{A}_{X}^{n}}$-module $\mathcal{F}$, such that $\left.\mathcal{F}\right|_{\mathbb{U}_{X}^{n}} \simeq \mathcal{E}_{X}^{(n)}$, then

$$
\left.\mathcal{E}_{k(x)}^{(n)} \simeq v_{f}^{*}\left(\mathcal{E}_{X}^{(n)}\right) \simeq v_{f}^{*}\left(\left.\mathcal{F}\right|_{\mathbb{U}_{X}^{n}}\right) \simeq \alpha_{f}^{*}(\mathcal{F})\right|_{\mathbb{U}_{k(x)}^{n}},
$$


and so it is enough to show the theorem for $X=\operatorname{Spec} R$ with $R$ a field. Similarly, localizing $R\left[T_{1}, \ldots, T_{n}\right]$ at the origin, we are reduced to the local case which follows from the following result of commutative algebra.

Theorem A.2. Let $(A, \mathfrak{m})$ be a regular local ring, $\underline{T}=\left(T_{1}, \ldots, T_{n}\right)$ be a regular system of parameters (see [7, Definition 2.2.1]), and

$$
\mathbb{U}=\bigcup_{i=1}^{n} \operatorname{Spec} A_{T_{i}}=\operatorname{Spec} A \backslash\{\mathfrak{m}\}
$$

be the punctured spectrum of $A$. Assume that $\operatorname{dim} A=n \geqslant 3$. Then

$$
\mathcal{S}_{j}:=\left.\operatorname{Ker}\left(K_{j}(A, \underline{T}) \stackrel{d_{j}(A, \underline{T})}{\longrightarrow} K_{j-1}(A, \underline{T})\right)\right|_{\mathbb{U}}
$$

cannot be extended to a free $A$-module if $n>j \geqslant 2$.

In the following let $\mathcal{I}_{j}=\operatorname{Ker} d_{j}(A, \underline{T})$, that is, $\mathcal{S}_{j}=\left.\mathcal{I}_{j}\right|_{\mathbb{U}}$. Recall also that $(-)^{\vee}=\operatorname{Hom}_{A}(-, A)$. For the proof we need the following result.

Proposition A.3. (i) Let $j \geqslant 2$. Then the $A$-module $\mathcal{I}_{j}$ is reflexive, that is, the natural morphism can $: \mathcal{I}_{j} \rightarrow \mathcal{I}_{j}^{\vee \vee}$ is an isomorphism.

(ii) If $M$ and $N$ are finitely generated $A$-modules, such that $\left.\left.M\right|_{\mathbb{U}} \simeq N\right|_{\mathbb{U}}$, and both $\left.M\right|_{\mathbb{U}}$ and $\left.N\right|_{\mathbb{U}}$ are locally free, then $M^{\vee} \simeq N^{\vee}$.

Proof. By assumption, $\mathcal{I}_{j}$ is a second syzygy and so (i) is a consequence of $[\mathbf{8}$, Theorem 3.6]. For (ii), by [12, Theorem 6.9.17] there exists $c \geqslant 0$, such that the given isomorphism $\left.\left.M\right|_{\mathbb{U}} \stackrel{\simeq}{\rightrightarrows} N\right|_{\mathbb{U}}$ is the restriction of a morphism $\mathfrak{m}^{c} M \rightarrow N$. Therefore we can assume that there exists $g: M \rightarrow N$, such that $\left.g\right|_{\mathbb{U}}$ is an isomorphism, that is, Ker $g$ and Coker $g$ have finite length.

Now we use the following fact (see [7, Theorem 1.2.8]). Since $\operatorname{dim} A \geqslant 2$ and $A$ is regular (and hence in particular Cohen-Macaulay), we have $\operatorname{Ext}_{A}^{i}(G, A)=0$ for any finite length module $G$ and $i=0,1$.

This and the exact sequences

$0 \longrightarrow \operatorname{Ker} g \longrightarrow M \longrightarrow \operatorname{Im} g \longrightarrow 0$ and $0 \longrightarrow \operatorname{Im} g \longrightarrow N \longrightarrow$ Coker $g \longrightarrow 0$

give $M^{\vee} \simeq(\operatorname{Im} g)^{\vee} \simeq N^{\vee}$.

Proof of Theorem A.2. Assume that $P$ is a free $A$-module, such that $\left.P\right|_{\mathbb{U}} \simeq \mathcal{S}_{j}$. We have $\left.\mathcal{I}_{j}\right|_{\mathbb{U}} \simeq \mathcal{S}_{j}$, too, and so $\mathcal{I}_{j}^{\vee} \simeq P^{\vee}$ by Proposition A.3(ii). Part (i) of this proposition tells us that $\mathcal{I}_{j}$ is reflexive and hence $\mathcal{I}_{j} \simeq \mathcal{I}_{j}^{\vee \vee} \simeq P^{\vee \vee}$ is free. But this is impossible, because

$$
\operatorname{Tor}_{n-j}^{A}\left(\mathcal{I}_{j}, A / \mathfrak{m}\right) \simeq \operatorname{Tor}_{n}^{A}(A / \mathfrak{m}, A / \mathfrak{m}) \simeq A / \mathfrak{m} \neq 0
$$

(note that here we need $j<n=\operatorname{dim} A$ ). We have the required result. 


\section{Appendix B. Product and 4-periodicity}

We have used in this work the fact that the product commutes with the translation. This has not been established in [11]. For the sake of completeness we give here a proof but refer to [11] for unexplained notation and definitions.

To start with let $\mathcal{A}^{(0)}=\left(\mathcal{A}, \mathcal{D}_{\mathcal{A}}, \delta_{\mathcal{A}}, \varpi^{\mathcal{A}}\right)$ and $\mathcal{B}^{(0)}=\left(\mathcal{B}, \mathcal{D}_{\mathcal{B}}, \delta_{\mathcal{B}}, \varpi^{\mathcal{B}}\right)$ be triangulated categories with $\delta_{\mathcal{A}^{-}}$respectively $\delta_{\mathcal{B}^{-}}$exact duality (like, for example, $\mathbb{D}^{\mathrm{b}}\left(\mathrm{VB}_{X}\right)$ with the usual 1-exact duality as in the main part of this work). We denote the shift functor in these triangulated categories by $\Sigma_{\mathcal{A}}$ respectively $\Sigma_{\mathcal{B}}$ (to distinguish we do not use $X \mapsto X[1])$. A symmetric $i$-space in $\mathcal{A}^{(0)}$ is a pair $(X, \psi)$ consisting of an object $X \in \mathcal{A}$ and a symmetric $i$-form $\psi: X \rightarrow \Sigma_{\mathcal{A}}^{i} \mathcal{D}_{\mathcal{A}} X$ which is an isomorphism; the symmetry of an $i$-form reads $\Sigma_{\mathcal{A}}^{i} \mathcal{D}_{\mathcal{A}}(\psi) \cdot \varpi_{X}^{\mathcal{A}}=(-1)^{i(i+1) / 2} \delta_{\mathcal{A}}^{i} \cdot \psi$. As in the case of derived categories, if $(X, \psi)$ is a symmetric $i$-form then $\left(\Sigma_{\mathcal{A}}^{2} X, \Sigma_{\mathcal{A}}^{2}(\psi)\right)$ is a symmetric $(i+4)$-form.

Let $(F, \rho): \mathcal{A}^{(0)} \rightarrow \mathcal{B}^{(0)}$ be a duality-preserving functor, that is, $\rho: F \mathcal{D}_{\mathcal{A}} \stackrel{\simeq}{\rightarrow} \mathcal{D}_{\mathcal{B}} F$ is an isomorphism of functors satisfying some compatibility axioms. We will only use the following. Since $F$ is a covariant exact functor between triangulated categories, there exists a family of isomorphisms of functors $\theta^{(i)}: F \Sigma_{\mathcal{A}}^{i} \stackrel{\simeq}{\rightarrow} \Sigma_{\mathcal{B}}^{i} F$ $(i \in \mathbb{Z})$ which are related by the following formulas:

$$
\theta^{(i+j)}=\Sigma_{\mathcal{B}}^{i}\left(\theta^{(j)}\right) \cdot \theta_{\Sigma_{\mathcal{A}}^{j}}^{(i)}
$$

$(i, j \in \mathbb{Z})$. Then we have

$$
\mathcal{D}_{\mathcal{B}} \Sigma_{\mathcal{B}}^{-1}\left(\theta_{\Sigma_{\mathcal{A}}^{-1}}^{(1)}\right) \cdot \rho_{\Sigma_{\mathcal{A}}^{-1}}=\left(\delta_{\mathcal{A}} \delta_{\mathcal{B}}\right) \cdot \Sigma_{\mathcal{B}}(\rho) \cdot \theta_{\mathcal{D}_{\mathcal{A}}}^{(1)}
$$

(cf. [11, Definition 1.8]). These axioms are made such that if $(X, \psi)$ is a symmetric $i$-space in $\mathcal{A}^{(0)}$ then

is a symmetric $i$-space in $\mathcal{B}^{(0)}$.

$$
(F, \rho)_{*}(X, \psi):=\left(F X,\left(\delta_{\mathcal{A}} \delta_{\mathcal{B}}\right)^{i} \Sigma_{\mathcal{B}}^{i}\left(\rho_{X}\right) \cdot \theta_{\mathcal{D}_{\mathcal{A}} X}^{(i)} \cdot F(\psi)\right)
$$

Lemma B.1. Let $(X, \psi)$ be a symmetric $i$-space in $\mathcal{A}$. Then there is an isometry

$$
(F, \rho)_{*}\left(\Sigma_{\mathcal{A}}^{2} X, \Sigma_{\mathcal{A}}^{2}(\psi)\right) \stackrel{\simeq}{\longrightarrow} \Sigma_{\mathcal{B}}^{2}\left((F, \rho)_{*}(X, \psi)\right) .
$$

Proof. We claim that $\theta_{X}^{(2)}: F \Sigma_{\mathcal{A}}^{2} X \stackrel{\simeq}{\rightarrow} \Sigma_{\mathcal{B}}^{2} F X$ is an isometry, that is, we have to show that

$$
\begin{aligned}
\left(\delta_{\mathcal{A}} \delta_{\mathcal{B}}\right)^{i+4} & \Sigma_{\mathcal{B}}^{i+4}\left(\rho_{\Sigma_{\mathcal{A}}^{2} X}\right) \cdot \theta_{\mathcal{D}_{\mathcal{A}} \Sigma_{\mathcal{A}}^{2} X}^{(i+4)} \cdot F \Sigma_{\mathcal{A}}^{2}(\psi) \\
& =\left(\delta_{\mathcal{A}} \delta_{\mathcal{B}}\right)^{i} \Sigma_{\mathcal{B}}^{i+4} \mathcal{D}_{\mathcal{B}}\left(\theta_{X}^{(2)}\right) \cdot \Sigma_{\mathcal{B}}^{i+2}\left(\rho_{X}\right) \cdot \Sigma_{\mathcal{B}}^{2}\left(\theta_{\mathcal{D}_{\mathcal{A}} X}^{(i)}\right) \cdot \Sigma_{\mathcal{B}}^{2} F(\psi) \cdot \theta_{X}^{(2)} .
\end{aligned}
$$

We observe first that $\Sigma_{\mathcal{B}}^{2} F(\psi) \cdot \theta_{X}^{(2)}=\theta_{\Sigma_{\mathcal{A}}^{i} \mathcal{D}_{\mathcal{A}} X}^{(2)} \cdot F \Sigma_{\mathcal{A}}^{2}(\psi)$ since $\theta^{(2)}$ is a natural transformation. By using (B.1) three times we get (recall that $\Sigma_{\mathcal{A}} \mathcal{D}_{\mathcal{A}}=\mathcal{D}_{\mathcal{A}} \Sigma_{\mathcal{A}}^{-1}$, by definition)

$$
\theta_{\mathcal{D}_{\mathcal{A}} \Sigma_{\mathcal{A}}^{2} X}^{(i+4)}=\Sigma_{\mathcal{B}}^{i+3}\left(\theta_{\mathcal{D}_{\mathcal{A}} \Sigma_{\mathcal{A}}^{2} X}^{(1)}\right) \cdot \Sigma_{\mathcal{B}}^{i+2}\left(\theta_{\mathcal{D}_{\mathcal{A}} \Sigma_{\mathcal{A}} X}^{(1)}\right) \cdot \Sigma_{\mathcal{B}}^{2}\left(\theta_{\mathcal{D}_{\mathcal{A}} X}^{(i)}\right) \cdot \theta_{\Sigma_{\mathcal{A}}^{i} \mathcal{D}_{\mathcal{A}} X}^{(2)}
$$

and so (B.3) is equivalent to

$$
\Sigma_{\mathcal{B}}^{i+4}\left(\rho_{\Sigma_{\mathcal{A}}^{2} X}\right) \cdot \Sigma_{\mathcal{B}}^{i+3}\left(\theta_{\mathcal{D}_{\mathcal{A}} \Sigma_{\mathcal{A}}^{2} X}^{(1)}\right) \cdot \Sigma_{\mathcal{B}}^{i+2}\left(\theta_{\mathcal{D}_{\mathcal{A}} \Sigma_{\mathcal{A}} X}^{(1)}\right)=\Sigma_{\mathcal{B}}^{i+4} \mathcal{D}_{\mathcal{B}}\left(\theta_{X}^{(2)}\right) \cdot \Sigma_{\mathcal{B}}^{i+2}\left(\rho_{X}\right) .
$$


Hence the result follows from the following calculation:

$$
\begin{aligned}
\Sigma_{\mathcal{B}}^{i+3}\left(\Sigma_{\mathcal{B}}\right. & \left.\left(\rho_{\Sigma_{\mathcal{A}}^{2} X}\right) \cdot \theta_{\mathcal{D}_{\mathcal{A}} \Sigma_{\mathcal{A}}^{2} X}^{(1)}\right) \cdot \Sigma_{\mathcal{B}}^{i+2}\left(\theta_{\mathcal{D}_{\mathcal{A}} \Sigma_{\mathcal{A}} X}^{(1)}\right) \\
= & \left(\delta_{\mathcal{A}} \delta_{\mathcal{B}}\right) \Sigma_{\mathcal{B}}^{i+3}\left(\mathcal{D}_{\mathcal{B}} \Sigma_{\mathcal{B}}^{-1}\left(\theta_{\Sigma_{\mathcal{A}} X}^{(1)}\right) \cdot \rho_{\Sigma_{\mathcal{A}} X}\right) \cdot \Sigma_{\mathcal{B}}^{i+2}\left(\theta_{\mathcal{D}_{\mathcal{A}} \Sigma_{\mathcal{A}} X}^{(1)}\right) \\
= & \left(\delta_{\mathcal{A}} \delta_{\mathcal{B}}\right) \Sigma_{\mathcal{B}}^{i+4}\left(\mathcal{D}_{\mathcal{B}} \theta_{\Sigma_{\mathcal{A}} X}^{(1)}\right) \cdot \Sigma_{\mathcal{B}}^{i+2}\left(\Sigma_{\mathcal{B}}\left(\rho_{\Sigma_{\mathcal{A}} X}\right) \cdot \theta_{\mathcal{D}_{\mathcal{A}} \Sigma_{\mathcal{A}} X}^{(1)}\right) \\
= & \Sigma_{\mathcal{B}}^{i+4} \mathcal{D}_{\mathcal{B}}\left(\Sigma_{\mathcal{B}} \theta_{X}^{(1)} \cdot \theta_{\Sigma_{\mathcal{A}} X}^{(1)}\right) \cdot \Sigma_{\mathcal{B}}^{i+2}\left(\rho_{X}\right) \\
= & \Sigma_{\mathcal{B}}^{i+4} \mathcal{D}_{\mathcal{B}}\left(\theta_{X}^{(2)}\right) \cdot \Sigma_{\mathcal{B}}^{i+2}\left(\rho_{X}\right)
\end{aligned}
$$

since $\theta_{X}^{(2)}=\Sigma_{\mathcal{B}} \theta_{X}^{(1)} \cdot \theta_{\Sigma_{\mathcal{A}} X}^{(1)}$ by (B.1).

Assume now that we have a third triangulated category with duality, say $\mathcal{C}^{(0)}=\left(\mathcal{C}, \mathcal{D}_{\mathcal{C}}, \delta_{\mathcal{C}}, \varpi^{\mathcal{C}}\right)$, and a dualizing pairing [11, Definition 1.11]

$$
\otimes: \mathcal{A}^{(0)} \times \mathcal{B}^{(0)} \longrightarrow \mathcal{C}^{(0)}
$$

Example B.2. Let $X$ be a scheme and $Z \subseteq X$ a closed subset. Then the (derived) tensor product

$$
\otimes_{\mathcal{O}_{X}}: \mathbb{D}^{\mathrm{b}}\left(\mathrm{VB}_{X}\right) \times \mathbb{D}_{Z}^{\mathrm{b}}\left(\mathrm{VB}_{X}\right) \longrightarrow \mathbb{D}_{Z}^{\mathrm{b}}\left(\mathrm{VB}_{X}\right)
$$

is a dualizing pairing. Note that in this case $\delta_{\mathcal{A}}=\delta_{\mathcal{B}}=\delta_{\mathcal{C}}=1$.

Let $(X, \psi)$ be a symmetric $i$-space in $\mathcal{A}^{(0)}$ and $(Y, \phi)$ a symmetric $j$-space in $\mathcal{B}^{(0)}$. The left product $(X, \psi) \star_{l}(Y, \phi)$ is then defined by considering $X \otimes-$ as a dualitypreserving functor with the aid of a duality transformation $\mathcal{L}(\psi)$ which depends on $\psi$, that is, the left product $(X, \psi) \star_{l}(Y, \phi)$ of these spaces is by definition $(X \otimes-, \mathcal{L}(\psi))_{*}(Y, \phi)$. The right product $\star_{r}$ is defined analogously by making the functor $-\otimes Y$ duality preserving using the symmetric $j$-form $\phi$. Both products are related by the following isometry:

$$
(X, \psi) \star_{l}(Y, \phi) \simeq\left(\delta_{\mathcal{A}} \delta_{\mathcal{C}}\right)^{j} \cdot\left(\delta_{\mathcal{B}} \delta_{\mathcal{C}}\right)^{i} \cdot(-1)^{i j} \cdot\left((X, \psi) \star_{r}(Y, \phi)\right) .
$$

(see $[\mathbf{1 1}$, Theorem 2.9]). From this we easily deduce the following.

LEMma B.3. There is an isometry

$$
\left(\Sigma_{\mathcal{A}}^{2} X, \Sigma_{\mathcal{A}}^{2}(\psi)\right) \star_{l}(Y, \phi) \simeq(X, \psi) \star_{l}\left(\Sigma_{\mathcal{B}}^{2} Y, \Sigma_{\mathcal{B}}^{2}(\phi)\right),
$$

and the same is true for the right product.

Proof. From Lemma B.1 we get isometries

and

$$
(X, \psi) \star_{l}\left(\Sigma_{\mathcal{B}}^{2} Y, \Sigma_{\mathcal{B}}^{2}(\phi)\right) \simeq \Sigma_{\mathcal{C}}^{2}\left((X, \psi) \star_{l}(Y, \phi)\right)
$$

$$
\left(\Sigma_{\mathcal{A}}^{2} X, \Sigma_{\mathcal{A}}^{2}(\psi)\right) \star_{r}(Y, \phi) \simeq \Sigma_{\mathcal{C}}^{2}\left((X, \psi) \star_{r}(Y, \phi)\right) .
$$

Hence the lemma follows by applying (B.4) twice.

Acknowledgements. We would like to thank Manuel Ojanguren for useful references. We would further like to thank the referee for useful suggestions. 


\title{
References
}

1. R. BAezA, Quadratic forms over semilocal rings (Springer, Berlin, 1978).

2. P. Balmer, 'Triangular Witt groups. Part I: the 12-term localization exact sequence', K-Theory 19 (2000) 311-363.

3. P. Balmer, 'Triangular Witt groups. Part II: from usual to derived', Math. Z. 236 (2001) $351-382$.

4. P. Balmer, 'Witt cohomology, Mayer-Vietoris, homotopy invariance, and the Gersten conjecture', K-Theory 23 (2001) 15-30.

5. P. BALmer, 'Vanishing and nilpotence of locally trivial symmetric spaces over regular schemes', Comment. Math. Helv. 78 (2003) 101-115.

6. P. Balmer and C. Walter, 'A Gersten-Witt spectral sequence for regular schemes', Ann. Sci. École Norm. Sup. (4) 35 (2002) 127-152.

7. W. Bruns and J. Herzog, Cohen-Macaulay rings (Cambridge University Press, 1993).

8. G. Evans and P. Griffith, Syzygies (Cambridge University Press, 1985).

9. S. Gille, 'Homotopy invariance of coherent Witt groups', Math. Z. 244 (2003) 211-233.

10. S. Gille, 'A transfer morphism for Witt groups', J. reine angew. Math. 564 (2003) 215-233.

11. S. Gille and A. Nenashev, 'Pairings in triangular Witt theory', J. Algebra 261 (2003) 292-309.

12. A. Grothendieck and J. Dieudonné, Eléments de géométrie algébrique I (Springer, Berlin, 1971).

13. M. Karoubi, 'Localisation de formes quadratiques II', Ann. Sci. École Norm. Sup. (4) 8 (1975) 99-155.

14. M. Knebusch, 'Symmetric bilinear forms over algebraic varieties', Conference on Quadratic Forms, Kingston, 1976 (ed. G. Orzech), Queen's Papers in Pure and Applied Mathematics 46 (Queen's University, Kingston, ON, 1977) 103-283.

15. M. OJAnguren and I. Panin, 'The Witt group of Laurent polynomials', Enseign. Math. 46 (2000) 361-383.

16. A. RAnicki, 'Algebraic L-theory', Comment. Math. Helv. 49 (1974) 137-167.

17. W. Scharlau, Quadratic and hermitian forms (Springer, Berlin, 1985).

18. C. Walter, 'Grothendieck-Witt groups of triangulated categories', Preprint, 2003, http://www.math.uiuc.edu/K-theory/0643/.

\author{
Paul Balmer and Stefan Gille \\ D-Math \\ ETH Zentrum \\ 8092 Zürich \\ Switzerland \\ balmer@math.ethz.ch \\ gille@math.ethz.ch
}

